\title{
Arp2/3 complex activity is necessary for mouse ESC differentiation, times formative pluripotency, and enables lineage specification
}

\author{
Francesca M. Aloisio and Diane L. Barber*
}

eTOC

Aloisio and Barber report a role for Arp2/3 complex activity in morphological and transcriptional changes with mESC differentiation. The authors reveal that inhibiting Arp2/3 complex activity delays entry into formative pluripotency, offering further mechanistic insight on this recently identified transition. Global examination of lineage specification using RNA-sequencing suggests a role for Arp2/3 complex activity in TBX3-dependent transcriptional programs during differentiation.

\section{Highlights}

- Arp2/3 complex activity is necessary for morphology changes during differentiation

- Arp2/3 complex activity regulates transcriptional markers of differentiation

- Inhibiting Arp2/3 complex activity delays entry into formative pluripotency

- Arp2/3 complex activity-dependent shuttling of FHL2 and MRTF occurs in mESCs 


\title{
Arp2/3 complex activity is necessary for mouse ESC differentiation, times formative pluripotency, and enables lineage specification
}

\author{
Francesca M. Aloisio and Diane L. Barber*
}

Department of Cell \& Tissue Biology; University of California San Francisco; San Francisco, CA, 94143; USA

*To whom correspondence should be addressed

Diane L. Barber

Box 0512

513 Parnassus Ave.

San Francisco, CA 94143

diane.barber@ucsf.edu

\section{Summary}

Mouse embryonic stem cells (mESCs), a model for differentiation into primed epiblast-like cells (EpiLCs), have revealed transcriptional and epigenetic control of early embryonic development.

The control and significance of morphological changes, however, remain less defined. We show marked changes in morphology and actin architectures during differentiation that depend on Arp2/3 complex but not formin activity. Inhibiting Arp2/3 complex activity pharmacologically or genetically does not block exit from naive pluripotency but attenuates increases in EpiLC markers. We find that inhibiting Arp2/3 complex activity delays formative pluripotency and causes globally defective lineage specification as indicated by RNA-sequencing, with significant effects on TBX3-depedendent transcriptional programs. We also identify two previously unreported indicators of mESC differentiation; MRTF and FHL2, which have inverse Arp2/3 complex-dependent nuclear translocation. Our findings on Arp2/3 complex activity in differentiation and the established role of formins in EMT indicate that these two actin nucleators regulate distinct modes of epithelial plasticity.

Keywords: mouse embryonic stem cells, actin cytoskeleton, morphology, pluripotency, differentiation, lineage specification, Arp2/3 complex, TBX3, MRTF, FHL2 


\section{Introduction}

As an in vitro model, mouse embryonic stem cells (mESCs) derived from the inner cell mass have provided insights on the regulated transition to primed pluripotent epiblast-like cells (EpiLCs) of the post-implantation blastocyst (Martin, 1981; Evans and Kaufman, 1981), which is one of the earliest known transitions in embryonic differentiation (Nichols and Smith, 2009; Nichols and Smith, 2012; Weinberger et al., 2016). While in vitro studies with mESCs have revealed how biochemical cues, transcriptional programs, and epigenetics drive differentiation, less is known about morphological changes during differentiation, how they are controlled, and their importance for naive cell differentiation or lineage specification (Gilmour et al., 2017; Villeneuve and Wickström, 2021).

Actin remodeling is a major driver of morphological changes that facilitates diverse cell behaviors (May, 2001; Goley and Welch, 2006). Actin filament architectures are predominantly generated by two classes of actin nucleators: the Arp2/3 complex comprising seven subunits that nucleates branched actin filaments, and formins, a family of fifteen mammalian isoforms that nucleate unbranched actin filaments. While neither class of actin nucleator has been reported for roles in pluripotency transition or lineage specification, processes they regulate, including cellular stiffness (Bongiorno et al., 2018), formation of ventral cortex F-actin asters (Xia et al., 2019), and apparent membrane tension (De Belly et al, 2021), and membrane-tocortex attachment (Bergert, Lembo et al., 2021), have roles in mESC differentiation. Here we show that mESC morphological changes and actin filament remodeling are dependent on activity of the Arp2/3 complex but not formins, and that Arp2/3 complex activity is necessary for transition from naive mESCs to EpiLCs, including timing entry into intermediate formative pluripotency with global effects on lineage specification.

Formative pluripotency, a recently identified intermediate stage during differentiation of naive mESCs to EpiLCs (Kalkan and Smith, 2014), is considered an executive phase when naive self-renewing signaling networks are dismantled and cells acquire competence for lineage 
specification. While the formative pluripotent state occurs during spontaneous mESC differentiation, a major limitation for a mechanistic understanding of formative pluripotency timing and regulation is the ambiguity of experimentally isolating and continuously propagating formative pluripotent cells (Smith, 2017; Kalkan et al., 2017; Mulas et al., 2017; Kinoshita and Smith, 2018). Our findings reveal a previously unrecognized role for the Arp2/3 complex in timing entry into formative pluripotency and subsequent lineage specification, which identifies new approaches for studying pluripotency transition states that could be applicable for regenerative medicine. Additionally, our work identifies opposing nuclear localization of myocardin-related transcription factor (MRTF) and FHL2, which are competing co-factors for serum response factor (SRF) transcriptional activity, as previously unreported markers of mESC differentiation and regulated by Arp2/3 complex activity.

\section{Results}

\section{Inhibiting Arp2/3 complex but not formin activity blocks morphological changes and actin remodeling during $\mathrm{mESC}$ differentiation}

A mechanism for changes in mESC morphology by actin remodeling and how they are regulated in the context of differentiation and lineage specification remains incompletely understood. Recent advances proposed roles for dynamic cell membrane tension and polarity as requisite regulators of both in vitro mESC differentiation (Xia et al., 2019; Bergert, Lembo et al., 2021) and in vivo embryonic development (Molé et al., 2021); however, to our knowledge a direct link between actin-dependent changes in morphology and the transcriptional programming of lineage specification has not been reported. To quantify changes in mESC colony morphology in real time we used quantitative DIC imaging of naive E14 mESCs maintained in the presence of LIF2i (Leukemia Inhibitory Factor and pharmacological inhibitors PD0325901 for MEK and CHIR99021 for glycogen synthase kinase-3ß) and spontaneously differentiated for 72h after removal of LIF2i (Ying et al., 2008). Naive colonies in LIF2i have a 
static circular morphology as quantified using circularity $=4$ pi(area/perimeter $\left.{ }^{\wedge} 2\right)$ with a value of 1.0 indicating a perfect circle and values approaching 0.0 indicating an elongated polygon shape (Fig. 1A-B, Supplemental Movie 1). In control cells, colony circularity progressively decreases at 24h, 48h and 72h after removing LIF2i (Fig. 1B, Supplemental Movie 2). Similar morphological changes during mESC differentiation were recently reported (Bongiorno et al., 2018; Bergert, Lembo et al., 2021); however, a regulatory mechanism for dynamic cell and colony morphology was not identified. In determining how these morphological changes are regulated we find that decreases in colony circularity at $48 \mathrm{~h}$ and $72 \mathrm{~h}$-LIF2i are significantly attenuated by CK666, a selective pharmacological inhibitor of Arp2/3 complex activity (Nolen et al., 2009; Yang et al., 2012) but not by SMIFH2, a broad-spectrum inhibitor of formin activity (Rizvi et al., 2009; Ganguly et al., 2015) (Fig. 1A-B). In contrast, we recently showed that SMIFH2 but not CK666 blocks morphological changes during epithelial to mesenchymal transition (EMT) (Rana et al., 2018). Although the selectivity of SMIFH2 was recently shown to also include non-muscle myosins (Nishimura et al., 2021), its ability to inhibit formin activity remains undisputed. Hence, our current findings indicate that the Arp2/3 complex and formins have distinct roles in morphological changes during two different programs of epithelial plasticity, mESC differentiation and EMT, respectively.

With the established role of Arp2/3 complex in nucleating branched actin filaments, we analyzed actin architectures during differentiation. Using high resolution spinning disc confocal imaging of phalloidin-labeled actin filaments we find that naive E14 mESCs in LIF2i have a compact polygonal cell shape with a cortical ring of actin filaments that are remodeled to an elongated cell shape with prominent membrane protrusions containing ribbed, fan-like actin filaments after 72h -LIF2i (Fig. 1C). In the presence of CK666 but not SMIFH2 actin filaments retain a cortical ring after $72 \mathrm{~h}$-LIF2i and fan-like filament networks are rarely seen (Fig. $1 \mathrm{C})$. We also find that effects with CK666 are phenocopied with CRISPR/Cas9 knockdown of ARPC2, an Arp2/3 complex subunit. We confirmed CRISPR/Cas9 editing of the Arpc2 locus by decreased 
ARPC2 in E14 mESCs by immunoblotting (Supplemental Fig. 1A-B) and by sequencing (Supplemental Fig. 1E-F). Consistent with the stability of Arp2/3 complex subunits being dependent on their assembly (Di Nardo et al., 2005; Steffen et al., 2006; Rauhala et al., 2013; LeClaire et al., 2015), with Arpc2 silencing in mESCs there is significantly decreased abundance of the Arp2/3 complex subunit ARP2 (Supplemental Fig. 1C-D). Inhibiting Arp2/3 complex activity in E14 mESCs by either CK666 or Arpc2 knockdown has no effect on the morphology or cortical actin organization of naive cells, but blocks pronounced fan-like actin filaments seen in membrane protrusions after 72h -LIF2i in control E14 cells (Fig. 1C). Similar to our findings with E14 mESCs, we confirmed that genetically distinct V6.5 mESCs show a similar remodeling of actin filament architectures with spontaneous differentiation that is blocked by CK666 but not SMIFH2 (Fig. 1D). Taken together, these findings indicate that actin remodeling with distinct changes in filament architectures occur during mESC differentiation in two mESC lines that are blocked by inhibiting Arp2/3 complex but not formin activity.

Changes in cell morphology are often not driven by actin filament remodeling alone but also in combination with actomyosin contractility (Murrell et al., 2015). In human induced pluripotent cells (iPSCs), a contractile actin fence promotes pluripotency and in mouse embryos suppressing actomyosin contractility regulates epiblast morphogenesis during pre- to postimplantation (Närvä et al., 2017; Molé et al., 2021). Consistent with our finding that CK666 attenuates changes in mESC colony morphology, immunolabeling indicates that phosphorylated MLC (pMLC), an indicator of actomyosin contractility, decorates the cortical actin ring around cells and the peripheral ring around free margins of colonies in control naïve mESCs but is diffuse in the cytoplasm after 72h -LIF2i (Fig. 1E). In contrast, in the presence of CK666 but not SMIFH2 pMLC retains a cortical localization after 72h -LIF2i (Fig. 1E). However, there is no change in pMLC abundance during differentiation in controls or with CK666 or SMIFH2, determined by immunoblotting of E14 mESC lysates (Supplemental Fig. 1G-H). Taken together, these data indicate that activity of the Arp2/3 complex but not formins regulates changes in 
colony morphology, actin architectures, and localized actomyosin contractility during mESC differentiation.

\section{Inhibiting Arp2/3 complex but not formin activity impairs differentiation to EpiLCs}

We used several approaches to show that Arp2/3 complex activity is also necessary for transcriptional changes during mESC differentiation. We first used a V6.5 dual-reporter (DR) mESC line engineered to express distinct fluorophores as cells transition from naive to primed pluripotency. In brief, work by Parchem et al. (2014) found that V6.5 mESCs in LIF2i express a naive-specific miR-290 cluster and with spontaneous differentiation upon removal of LIF2i miR290 expression decreases and expression of the primed-specific miR-302 cluster increases. They generated cells that express mCherry driven by the miR-290 promoter and GFP driven by the miR-302 promoter. Using flow cytometry, DR mESCs can be used to score for decreased mCherry expression and increased GFP expression as an index of differentiation on the cell population level, while intermediate cells are double-positive for both markers (Fig. 2A). Our analysis indicates that control DR mESCs in LIF2i are $>90 \%$ mCherry positive (Supplementary Fig. $2 \mathrm{~A}$ ), which is reduced to $16.3 \%$ after $72 \mathrm{~h}$-LIF2i in controls but is significantly greater at 44.0\% with CK666 (Fig. 2B). In contrast, the percent of mCherry single-positive cells is not different with CK689, an inactive analog of CK666 (Nolen et al., 2009), the formin inhibitor SMIFH2, or DMSO as a vehicle compared with controls (Fig. 2B). Further, cell death and proliferation in the presence of CK666, CK689, SMIFH2 or DMSO are not significantly different from control cells at any timepoint during differentiation (Supplementary Fig. 2B, 2E). These data indicate that Arp2/3 complex but not formin activity is necessary for changes in stagespecific miRNA expression during naive to primed pluripotency, suggesting a broader role for Arp2/3 complex-dependent actin remodeling in the context of mESC differentiation beyond changes in morphology. 
As a second approach to test differentiation, we confirmed that CK666 and Arpc2 silencing attenuates expression of established primed EpiLC markers. RT-qPCR for Fgf5 (Fig. 2C) and Brachyury (Fig. 2D) in V6.5 DR mESCs indicates significantly increased expression in controls and with CK689 after 72h -LIF2i but not with CK666. We used a similar approach to show that expression of Fgf5 in E14 mESCs significantly increases after 72h -LIF2i in controls but is attenuated with Arpc2 silencing (Fig. 2E). These data support a role for Arp2/3 complex activity in transcriptional changes associated with differentiation of naive to primed EpiLCs as indicated by pharmacologically or genetically inhibiting Arp2/3 complex activity in V6.5 and E14 mESCs.

Our third approach to test differentiation scored for the cytosolic and nuclear localization TFE3, a bHLH transcription factor that is predominantly nuclear in naive mESCs but mostly cytoplasmic in primed EpiLCs (Betschinger et al., 2013; Villegas et al., 2019; Kalkan et al., 2017). Using quantitative immunolabeling of E14 mESCs, we see that the nuclear to cytoplasmic ratio of endogenous TFE3 significantly decreases after $72 \mathrm{~h}$-LIF2i in controls but not in the presence of CK666 (Fig. 2F-G). Taken together, these data reveal a role for Arp2/3 complex activity beyond morphology to include transcriptional indicators such as miRNA expression, primed marker gene expression, and transcription factor localization during transition of naive mESCs to primed EpiLCs.

\section{Inhibiting Arp2/3 complex activity has no effect on exit from naïve self-renewal but delays entry into formative pluripotency}

To further understand how Arp2/3 complex activity enables mESC differentiation, we tested whether it is necessary for exit from naive pluripotency. At 72h -LIF2i, the naive marker Rex1 (also called Zfp42) significantly decreases in V6.5 and E14 cells in the absence and presence of CK666 and CK689 (Fig. 3A-B) as well as in E14 cells with Arpc2 silencing (Fig. 3B). Moreover, the time-dependent decrease in the expression of Rex1 as well as Stra8, an additional naive 
marker, over 120h -LIF2i in E14 cells is similar in the absence or presence of CK666 (Fig. 3C, Supplemental Fig. 2F). These data suggest that although Arp2/3 complex activity is necessary for increased primed EpiLC markers seen in controls, it is not necessary for maintaining naive markers or for exit from naive pluripotency.

An intermediate state between naive and primed pluripotency, termed formative pluripotency, was recently identified. Formative pluripotency is considered an "executive" state when cells are most responsive to differentiation cues and most receptive for lineage commitment (Smith, 2017; Kalkan et al., 2017; Kalkan et al., 2019). Given our findings that inhibiting Arp2/3 complex activity has no effect on exit from naïve pluripotency but attenuates markers of primed pluripotency, we tested whether inhibiting Arp2/3 complex affects expression of intermediate formative pluripotent markers. The formative pluripotent state is currently defined by decreased expression of Rex 1 , which we confirmed is not impaired when Arp2/3 activity is inhibited (Fig. 3A-C), increased Otx2 (Kalkan et al., 2017; Mulas et al., 2017), increased phosphorylated ERK (pERK) (Kalkan et al., 2019), and increased Grh/2 (Chen et al., 2018). We confirmed that Otx2 significantly increases in control E14 cells within 24h -LIF2i (Fig. 3D). In contrast, with CK666 Otx2 expression at 24h -LIF2i is significantly less compared with control cells and not different than in naive cells (Fig. 3D). After 48h -LIF2i, however, CK666treated cells have a delayed increase in Otx2 (Fig. 3D). These results are temporally consistent both with the delayed decrease in colony circularity observed in CK666-treated E14 cells at 48h -LIF2i (Fig. 1A-B) and with previous reports for delayed Otx2 expression at 48h -LIF2i in the presence of a pharmacological inhibitor of NODAL signaling, which is suggested to function as a timing mechanism for pluripotency transition (Mulas et al., 2017).

Increased pERK, another marker of formative pluripotency, is required for activating downstream formative pluripotent gene regulatory networks (Kalkan et al., 2019; Azami et al., 2019). We find increased pERK in control E14 cells at 24 and $48 \mathrm{~h}$-LIF2i compared with total ERK, which does not change during differentiation, as determined by immunoblotting cell 
lysates (Fig. 3E-F). In contrast, with CK666 pERK does not increase in -LIF2i cells compared with naive cells (Fig. 3E-F). We also used immunoblotting of E14 cell lysates to confirm increased abundance of GRHL2 in control E14 cells at 24 and 48h -LIF2i (Fig. 3G-H), which is similar to reported findings using V6.5 cells (Chen et al., 2018). In contrast, with CK666 increased GRHL2 is delayed with a significant increase at $48 \mathrm{~h}$ but not at $24 \mathrm{~h}$ in -LIF2i in cells (Fig. 3G-H). Further, expression of Cldn6, a downstream target gene of GRHL2 in mESCs, significantly increases in control E14 cells at 24h -LIF2i but not with CK666 (Fig. 3I). Hence, inhibiting Arp2/3 complex activity in two different mESC lines has no effect on maintenance of naive self-renewal or exit from naive pluripotency but delays entry into the intermediate formative pluripotent stage as indicated by attenuated Otx2 and Cldn6 expression as well as pERK and Grhl2 abundance.

\section{Inhibiting Arp2/3 complex activity disrupts lineage commitment with pronounced effects on TBX3 target genes across all three germ layers}

Our findings that Arp2/3 complex activity is necessary for actin remodeling, attenuated expression of primed marker expression, and timing for formative pluripotency during mESC differentiation suggest a role in promoting primed EpiLC lineage specification. To investigate global effects of inhibiting Arp2/3 complex activity on lineage specification, we performed RNA sequencing (RNA-seq) on E14 cells differentiated in the absence and presence of CK666 for 72h -LIF2i and control naive cells maintained in LIF2i (Fig. 4A-B). We found that control naïve +LIF2i and -LIF2i cells have a total of 6,576 differentially expressed genes (DEGs) with an adjusted qval $<0.05$ after batch correction (Fig. 4C). Of these DEGs, 1,662 are unique to control -LIF2i cells compared with naïve +LIF2i cells and are not differentially expressed in CK666 -LIF2i compared with naïve +LIF2i cells (Fig. 4C). CK666 -LIF2i cells compared with naïve +LIF2i have 4,796 DEGs, with 457 unique DEGs (Fig. 4C). In CK666 -LIF2i cells compared with both control +/-LIF2i cells, 972 unique DEGs are displayed (Fig. 4A). Gene 
Ontology (GO) enrichment analysis of this latter subset suggests that unique CK666-specific DEGs are associated with biological processes related to extracellular matrix organization, endothelial cell migration, sprouting angiogenesis, and the MAPK/ERK cascade (Fig. 4D). As a general summary, these data indicate global transcriptomic differences in naive +LIF2i cells, control -LIF2i cells, and CK666-treated -LIF2i cells.

Consistent with our data indicating that CK666 has no effect on exit from naïve selfrenewal (Fig. 3A-C), RNA-seq data also show downregulated naive markers in the presence of CK666 compared with control cells with the exception of Tbx3 (Fig. 4E). TBX3 is a master regulatory transcription factor known to play dual inhibitory and activating roles as mESCs transition from naive self-renewal to lineage specification (Lu et al., 2011; Kalkan et al., 2019). Consistent with significantly attenuated Tbx3 expression in CK666 -LIF2i, a number of TBX3 target genes (Russell et al., 2015; Nishiyama et al., 2013; Han et al., 2010) such as Fgf5, Fn1, Zeb1, Kctd12b, and Mat2a (Fig. 4B) and targets specific to mesoderm such as Pdlim3, Adm, and Fhl2, (Fig. 4F), endoderm such as Eomes and Kit, (Fig. 4G), and ectoderm such as Mycn, Prickle1, and Nes (Fig. 4H) are significantly dysregulated. Taken together, these data indicate a role for Arp2/3 complex activity in timing of formative pluripotent lineage specification related to delayed extinction of naive-promoting targets of $T b \times 3$, which is suggested to counteract the initiation of formative pluripotent gene regulatory networks (Kalkan et al., 2019).

TBX3 is an established regulator of early development with dynamic context-dependent roles in embryonic organogenesis across germ layers (Chapman et al., 1996). With its binding to a number of transcription factors such as Klf4, Oct4, Sox2, and Nanog, TBX3 plays a complex role at the center of pluripotency circuitry (Han et al., 2010; Russell et al., 2015) with the potential to act as either an activator or inhibitor of gene expression dependent upon cofactor binding (Carlson et al., 2001). To determine the extent to which inhibiting Arp2/3 complex activity globally affects TBX3 target gene expression, we compared our DEGs to three publicly available mESC datasets related to target genes that change expression relative to a 
TBX3 reporter (Fig. 5A) (Russell et al., 2015), change expression with shRNA knockdown of Tbx3 (Fig. 5B) (Nishiyama et al., 2013), and bind TBX3 as indicated by ChIP-sequencing (Fig. 5C) (Han et al., 2010). Comparing data sets shows that CK666 treatment during differentiation generally causes TBX3 target genes to have a contrasting transcriptional profile compared with that of a control differentiation: for each TBX3 gene list, our clustermaps indicate that genes with increasing expression in control -LIF2i had attenuated expression with CK666 and genes with decreasing expression in control -LIF2i had heightened expression with CK666 (Fig. 5A-C). Enriching for TBX3 target genes common to all three datasets that are significantly dysregulated with CK666 compared with control (Fig. 5D) suggests effects on a number of key regulators such Mycn, which is essential for neurogenesis (Knoepfler et al., 2002; Kerosuo et al., 2018), Prdm1, which has multiple roles in neural fate and germ cell specification (Prajapati et al., 2019; Ohinata et al., 2005), and Cobl, which has an actin-related role in neural tube formation (Carroll et al., 2003). Other noteworthy gene expression changes include Tfe3 (Fig. 2F-G, Fig. 5A) and Cldn6 (Fig. 3I, Fig. 5A). Additionally, Eomes, a TBX3 target gene that plays a contextdependent role in specification of all three germ layers (Costello et al., 2011; Tosic et al., 2019) and Fh/2, a mesodermal marker and recently identified tension-dependent actin-binding protein (Sun et al., 2020), are significantly attenuated in the presence of CK666 (Fig. 4F, 5A). Collectively, these data suggest that Arp2/3 complex activity times entry into formative pluripotency, possibly by delayed loss of $T b x 3$ expression, resulting in defective downstream global and distinct lineage specification programs.

\section{Inhibiting Arp2/3 complex activity blocks cytoplasmic and nuclear shuttling of MRTF and FHL2}

Our findings on TBX3 target genes regulated by Arp2/3 complex activity led us to identify two previously unreported markers of mESC differentiation - the cytoplasmic and nuclear localization of SRF co-transcriptional activators FHL2 and MRTF. MRTF is an actin 
polymerization-responsive transcriptional co-activator that, with increased actin polymerization, translocates to the nucleus (Miralles et al., 2003; Posern and Treisman, 2006). FHL2 is a TBX3 target gene and a transcriptional co-activator that is predominantly nuclear in response to decreased F-actin tension (Philippar et al., 2004; Nakazawa et al., 2016). Although formindependent nuclear translocation of MRTF is well-described for adult mesenchymal stem cell differentiation, neither MRTF nor FHL2 translocation has been reported to be regulated by Arp2/3 complex activity nor to translocate during mESC differentiation.

We scored for changes in MRTF localization during mESC differentiation and found that in control and SMIFH2-treated naive E14 cells MRTF is diffuse in the cytoplasm but after $72 \mathrm{~h}$ LIF2i becomes predominantly nuclear as quantified by a significant increase in the nuclear to cytoplasmic ratio (Fig. 6A, B). In contrast, with CK666 nuclear translocation of MRTF is inhibited with no increase in nuclear abundance at both 72h -LIF2i (Fig. 6A, B) and at 120h -LIF2i (Supplemental Fig. 3A, B). These data indicate that MRTF nuclear translocation occurs during mESC differentiation and is dependent on activity of the Arp2/3 complex but not formins. Further, our RNA-seq data confirm that MRTF target genes (Esnault et al., 2014) have a contrasting transcriptional profile with CK666 compared to controls: our clustermap indicates that MRTF target genes going up in control -LIF2i had attenuated expression with CK666 and genes going down in in control -LIF2i had heightened expression with CK666, including Fh/2 and Srf (Fig. 6C).

Similar to MRTF, nuclear FHL2 binds with transcription factor SRF to promote target gene expression with noted functions in mesoderm tissues (Lorda-Diez et al., 2018; Renger et al., 2013; Esnault et al., 2014; Philippar et al., 2004; Russell et al., 2015). We scored for changes in FHL2 localization during mESC differentiation and found that in control naive E14 cells FHL2 is nuclear but after $72 \mathrm{~h}$-LIF2i undergoes translocation to the cytoplasm, quantified by a significant decrease in the nuclear to cytoplasmic ratio (Fig. 6D, E). In contrast, with CK666 cytoplasmic translocation of FHL2 is attenuated with no significant decrease in nuclear 
abundance at 72h -LIF2i (Fig. 6D, E). Taken together, these data reveal three previously unrecognized events during mESC differentiation; first is changes in the localization of MRTF and FHL2, second is the opposing nuclear and cytoplasmic translocation of these SRF transcriptional coactivators, and third is that their translocation is dependent on Arp2/3 complex activity (Fig. 6F).

\section{Discussion}

We report a previously unrecognized function of Arp2/3 complex activity in enabling the differentiation of naive to primed mESCs. We find that changes in colony morphology and actin architectures in mESCs are dependent on activity of the Arp2/3 complex but not formins. Our data also indicate that Arp2/3 complex activity is necessary for transition to distinct pluripotency states: although not necessary for exit from the naive state, loss of Arp2/3 complex activity delays entry into the formative pluripotent state, contributing further mechanistic insight on how this newly identified intermediate state is controlled (Smith, 2017; Kalkan et al., 2017; Kalkan et al., 2019). Further, our data include a global examination of actin-dependent lineage specification across all three germ layers in mESCs, which align with evolutionarily conserved roles for force-sensitive differentiation and development in other organisms both and in vitro (Chowdhury et al., 2010; Lee et al., 2013) and in vivo (Keller et al., 2003; Krieg et al., 2008). Lastly, we show for the first time that MRTF and FHL2, both actin-responsive transcriptional coactivators to SRF, undergo inverse Arp2/3 complex activity-dependent translocation events during mESC differentiation.

We show marked changes in colony morphology and actin architectures during differentiation that depend on Arp2/3 complex but not formin activity. These findings are consistent with reported observations related to morphology and dynamic cellular stiffness during differentiation (Bongiorno et al., 2018) and an acute role for the Arp2/3 complex in mESC actin remodeling (Xia et al., 2019). We observed Arp2/3 complex-dependent actin architectures, 
which are estabished to generate generate protrusive forces for membrane dynamics (Bailly et al., 2001; Swaney and Li, 2016) and is of particular interest with regard to recently reported roles for dynamic membrane tension related to cortical actin detachment during mESC pluripotency transition (Bergert, Lembo et al., 2021; De Belly et al., 2021). An important question to resolve is how Arp2/3 complex activity regulates transcriptional changes in mESCs compared with its regulation of transcriptional events in other cell models (Yoo et al., 2007; Olson and Nordheim, 2010). Previous studies have indicated force-sensitive lineage specification (Keller et al., 2003; Krieg et al., 2008; Gilmour et al., 2017; Villeneuve and Wickström, 2021) as well as mechanosensing and contractility in fate specification for mESCs (Janmey et al., 2013; Happe and Engler, 2016; Tatapudy et al., 2017). The role of Arp2/3 complex as a central node between biochemical cues and biophysical responses (Iskratsch et al., 2014; Charras and Yap, 2018) suggests a mechanosensitive mechanism whereby Arp2/3 complex activity enables mESC differentiation.

Our data also suggest that Arp2/3 complex activity times entry to intermediate formative pluripotency, an "executive" state when cells are most receptive for lineage specification cues (Smith, 2017; Kalkan et al., 2017; Kalkan et al., 2019). Inhibiting Arp2/3 complex activity delays entry into formative pluripotency, as indicated by the delayed increase in Otx2 and Cldn6 expression, and GRHL2 abundance as well as no change in pERK with -LIF2i compared with controls. In related findings, inhibiting NODAL signaling has no effect on exit from naive pluripotency but delays formative pluripotent marker expression from $24 \mathrm{~h}$ to $48 \mathrm{~h}$-LIF2i (Mulas et al., 2017). Taken together, our data indicate that Arp2/3 complex activity is a previously unrecognized node in the growing signaling network of formative pluripotent regulators, which adds mechanistic insight relevant to embryonic lineage specification.

We also reveal that inhibiting Arp2/3 complex activity disrupts lineage commitment across all three germ layers with pronounced effects in TBX3 target genes compared with control cells. TBX3 is a context-dependent master regulator of both naive self-renewal (Niwa et 
al., 2009; Han et al., 2010; Russell et al., 2015) and lineage specification (Costello et al., 2011; Lu et al., 2011; Weidgang et al., 2013; Kartikasari et al., 2013) and its continued expression during mESC differentiation is reported to destabilize entry into formative pluripotency (Kalkan et al., 2019). Dysregulated Tbx3 expression is associated with atypical cell and colony morphology in mESCs (Han et al., 2010; Russell et al., 2015), which we also see with loss of Arp2/3 complex activity. Smith and colleagues proposed a relationship between timing of formative pluripotency and RBPJ, a regulator of mESC morphology, whereby RBPJ inhibits TBX3 expression to block formative cells from returning to self-renewal (Kalkan et al., 2019). Further, ERK signaling is reported to inhibit Tbx3 expression and mESCs null for ERK pathway components have sustained Tbx3 expression (Niwa et al., 2009; Hamilton et al., 2013; Chen et al., 2015). Our data indicating attenuated pERK and persistent Tbx3 abundance with CK666 suggest a relationship between Arp2/3 complex activity, mESC morphology, formative pluripotency and TBX3-dependent lineage specification. Future studies on the link between Arp2/3 complex activity and formative pluripotency timing will be important to resolve the interface between morphology and lineage specification, with potential relevance to enhanced protocols for directed differentiation.

We also show that MRTF and FHL2, actin-responsive transcriptional co-activators for SRF, undergo previously unreported translocation events during mESC differentiation. MRTF is a transcriptional co-activator SRF (Posern and Treisman, 2006; Sun et al., 2006; Vartiainen et al., 2007). With increased actin polymerization, MRTF translocates from the cytoplasm to the nucleus where it binds to SRF to promote differentiation programs in adult mesenchymal stem cells (Miralles et al., 2003; Nobusue et al., 2014; McDonald et al., 2015; Bian et al., 2016). Our data show two previously unreported findings on MRTF nuclear translocation; first that it occurs with mESC differentiation and second that it is dependent on Arp2/3 complex activity. Although MRTF has not been reported for direct roles in embryonic differentiation, SRF is confirmed to regulate embryonic mesoderm formation (Weinhold et al., 2000) and Srf-/- ESCs have altered 
cell morphology and reduced cortical actin (Schratt et al., 2002). FHL2, dysregulated in many cancers and developmental disorders, is another SRF-binding transcriptional co-activator which exhibits a direct transcriptional response to the actin cytoskeleton (Sun et al., 2020) and is a TBX3 target gene (Russell et al., 2015). In the cytosol, FHL2 contains LIM domains that mechanoaccumulate on strain sites of tensed actin filaments (Sun et al., 2020). FHL2 is released from F-actin upon loss of filament strain, causing it to translocate to the nucleus where it competes with MRTF for SRF-binding (Philippar et al., 2004). In tandem, MRTF and FHL2 are both direct actin-responsive transcriptional co-activators with dueling roles both in the cytosol and in the nucleus: in the cytosol competing for actin-binding and in the nucleus competing for SRF-binding to promote distinct transcriptional programs. Our data show a previously unreported inverse translocation of MRTF and FHL2 with mESC differentiation. Taken together, these data suggest that inversely mechanosensitive translocation events of MRTF and FHL2 could serve as a novel marker for pluripotency status and provide motivation for understanding how basic cell biology such as actin remodeling can provide a framework for elucidating mechanisms of mESC differentiation and lineage specification.

As recently indicated (Gilmour, 2017; Villeneuve and Wickström, 2021), a current challenge is to identify the connection between the cellular machines that generate shape and the genes that control cell-fate decisions. Our observations, compared with previous findings that activity of formins but not Arp2/3 complex is necessary for EMT and the assembly of unbranched contractile actin filaments (Li et al., 2010; Jurmeister et al., 2012; Rana et al., 2018), indicate that these different classes of actin nucleators and the architectures they generate have selective roles in distinct types of epithelial plasticity. With known functions in migration (Suraneni et al., 2012; Arnold 2008) and adherens junction tension (Verma et al., 2012; Fierro-Gonzales et al., 2012), there are abundant potential mechanisms whereby Arp2/3 complex activity might regulate mESC pluripotency transition (Rotty et al., 2013; Pieters and van Roy, 2014; Wagh et al., 2021; Molé et al., 2021). Our study provides a step toward closing the 
gap between phenotype and genotype, opening new directions and advancing new approaches to understand how morphological changes and actin filament dynamics promote pluripotency transition, with potential value for additional approaches in directing differentiations for regenerative medicine.

\section{Materials and Methods}

\section{Cell culture}

Wild-type and DR V6.5 ESCs, obtained from R. Blelloch (University of California San Francisco), and E14 ESCs, provided by A. Smith (University of Cambridge) were maintained in tissue culture dishes coated with $0.2 \%$ gelatin (G1393; Sigma) at $37^{\circ} \mathrm{C}$ and $5 \% \mathrm{CO} 2$ in DMEM (10569; Gibco) supplemented with 15\% FBS (FB-11, Omega Scientific, Inc.), glutamine (2 mM), non-essential amino acids $(0.1 \mathrm{mM})$, penicillin-streptomycin $(100 \mathrm{U} / \mathrm{mL}$ Penicillium and 100 $\mu \mathrm{g} / \mathrm{mL}$ Streptomycin), and 2-mercaptoethanol (55 $\mu \mathrm{M})$. Cells received fresh medium every $24 \mathrm{~h}$ and were passaged every three days after dissociating with $0.25 \%$ Trypsin-EDTA (25200-056; Gibco). For self-renewal, cells were maintained in medium containing LIF (ESGRO Cat\#ESG1106; EMD Millipore) and inhibitors for MEK (1 $\mu$ M; PD0325901, Cat\#S1036; Selleck Chemicals) and glycogen synthase kinase-3ß (1 $\mu \mathrm{M}$; CHIR99021, Cat\#S2924; Selleck Chemicals), collectively termed LIF2i. To induce spontaneous differentiation cells were washed in PBS and then incubated in medium without LIF2i as described in Parchem et al. (2014) for the indicated times. CK666 (80 $\mu \mathrm{M}$ final; 182515; EMD Millipore), CK689 (80 $\mu \mathrm{M}$ final; 182517; EMD Millipore), and SMIFH2 (25 $\mu \mathrm{M}$ final; S4826; Sigma) were added at 1:000 from stock solutions prepared in DMSO at the indicated times and included in medium replacements every 24h. Mycoplasma contamination was tested 2x/year using media obtained from cells maintained for $48 \mathrm{~h}$ in the absence of penicillin-streptomycin by using a PCR Mycoplasma Detection Kit (abm Cat\#G238). Cell lines were authenticated commercially by IDEXX BioAnalytics (USA). 


\section{CRISPR/Cas9 gene editing}

The validated guide RNAs (gRNA) targeting the Arpc2 locus were selected from the Genomescale CRISPR Knock-Out (GeCKO) v2 mouse library (www.genome-engineering.org) (Sanjana et al., 2014). After annealing and adding Bbsl cut site overhangs, candidate gRNAs were cloned into the pSpCas9(BB)-2A-GFP (PX458) plasmid vector (Addgene plasmid \#48138; RRID: Addgene_48138) (Ran et al., 2013). At $48 \mathrm{~h}$ after transfecting cells with plasmids, single GFP(+) cells were sorted by fluorescence-activated cell sorting as described below. Edited clones were validated by PCR and sequencing (forward primer AGCTGTTGAATGCAATGAGG, reverse primer TCCTCTGGGTAAAGGACCT) and confirmed by immunoblotting as described below. TIDE webtool (https://tide.deskgen.com) was used to quantify editing efficacy and identify the predominant type of indel in the edited clone (Brinkman et al., 2014). The gRNA sequence used to generate the confirmed Arpc2 edited clone was as follows: TTCTTGGTAAATCCAGAACC.

\section{DIC image acquisition and quantitative analysis}

For DIC imaging, naïve E14 ESCs were plated for 24h on gelatin-coated glass bottom microwell dishes (P35G-1.5-14-C; MatTek) in medium containing LIF2i, washed with PBS, and then maintained for the indicated times in medium without LIF2i. CK666 and SMIFH2, as indicated above, were added at the time of LIF2i removal and replaced every $24 \mathrm{~h}$ until completion of imaging. Live cells were imaged using a Plan Apo 400.95 NA objective on an inverted spinning disc microscope system (Nikon Eclipse TE2000 Perfect Focus System; Nikon Instruments; Nikon Instruments) equipped with D-C DIC Slider 40x I (MBH76240; Technical Instruments), a multipoint stage (MS-2000; Applied Scientific Instruments), a CoolSnap HQ2 cooled chargecoupled camera (Photometrics) and camera-triggered electronic shutters controlled with NISElements Imaging Software (Nikon). Approximately 15-20 colonies were imaged for each condition and time point. Colony circularity was quantified using the ImageJ plug-in "Circularity" feature. In brief, this feature is an extended version of the Measure command in ImageJ that 
calculates object circularity using the formula circularity $=4$ pi(area/perimeter $\left.{ }^{\wedge} 2\right)$, with a circularity value of 1.0 indicating a perfect circle. As the value approaches 0.0 , it indicates an increasingly elongated polygon. Statistical analysis was performed with GraphPad Prism 6 software.

\section{Immunolabeling, staining, and image acquisition}

For immunolabeling, cells were plated on gelatin-coated coverslips prepared in an ultrasonic cleaning bath. In brief, coverslips were sonicated for 20 minutes in the presence of ddH2O and Versa detergent, washed in $\mathrm{ddH} 2 \mathrm{O}$, sonicated again for 20 minutes, and stored in $70 \% \mathrm{EtOH}$. Cells were maintained for the indicated times, washed with PBS, and fixed with $4 \%$ formaldehyde for $15 \mathrm{~min}$ at RT. Cells were then permeabilized with $0.1 \%$ Triton $\mathrm{X}-100$ for $5 \mathrm{~min}$, incubated with blocking buffer of $5 \%$ horse serum and $1 \%$ BSA in PBS for $1 \mathrm{~h}$, and then incubated with primary antibodies overnight at $4^{\circ} \mathrm{C}$. The cells were then washed with PBS, incubated for $1 \mathrm{~h}$ at RT with secondary antibodies conjugated with fluorophores, and washed with PBS. One wash included Hoechst 33342 (1:10,000; H-3570; Molecular Probes) to stain nuclei. Primary antibodies included Phospho-Myosin Light Chain 2 Thr18/Ser19 E2J8F (1:200; \#95777; Cell Signaling Technology), MRTF-A-C19 (1:200; sc-21558; Santa Cruz Biotechnologies), FHL2 (1:200; HPA006028; Sigma), and TFE3 (1:200; 14480-1-AP; Proteintech). Actin filaments were labeled with rhodamine-phalloidin (1:400; Invitrogen) added during secondary antibody incubations. Cells were imaged using a 60X Plan Apochromat TIRF 1.45 NA oil immersion objective on an inverted microscope system (Nikon Eclipse TE2000 Perfect Focus System; Nikon Instruments) equipped with a spinning-disk confocal scanner unit (CSU10; Yokogawa), a 488-nm solid-state laser (LMM5; Spectral Applied Research), a multipoint stage (MS-2000; Applied Scientific Instruments), a CoolSnap HQ2 cooled chargecoupled camera (Photometrics) and camera-triggered electronic shutters controlled with NISElements Imaging Software (Nikon). Nuclear-to-cytoplasmic ratios were quantified using NIS- 
Elements Imaging Software (Nikon). Briefly, the fluorescence in the nucleus and cytoplasm were manually sampled by selection of regions-of-interest either colocalized with nuclear DAPI or not. The ratio of fluorescence was then calculated by dividing the nuclear fluorescence intensity with that of the cytoplasm for a given cell. Statistical analysis was performed in Excel (Microsoft) using two-tailed t-test.

\section{Flow Cytometry}

DR ESCs and CRISPR-Cas9 edited E14 ESCs were prepared for flow cytometry by washing with PBS at the indicated times, dissociated with $0.25 \%$ Trypsin-EDTA, and collected by centrifuging at $1000 \mathrm{rpm}$ for $3 \mathrm{~min}$ at room temperature. Pelleted cells were washed in cold PBS, pelleted again by centrifugation, and then resuspended to a final concentration of 5-10 $\mathrm{x} 10^{6} \mathrm{cells} / \mathrm{ml}$ in PBS supplemented with $1 \%$ BSA. Cell suspensions were filtered into roundbottomed tubes with cell-strainer caps (352235; Falcon). DR ESCs were sorted using an LSR II flow cytometer (BD Biosciences) and CRISPR-Cas9 edited E14 ESCs were sorted using FACSAria III flow cytometer (BD Biosciences), and analysis was performed using FACSDiva software (BD Biosciences). Statistical analysis was performed in Excel (Microsoft) using twotailed t-test.

\section{RNA extraction, cDNA synthesis, and qPCR}

Total RNA was isolated from ESCs at the indicated times by using TRIzol Reagent (15596026; Ambion) according to the manufacturer's protocol with the following modifications: after washing cells with PBS $800 \mu \mathrm{l} \mathrm{TRIzol}$ was added to cells in a six-well plate and the pellet was rinsed in $75 \% \mathrm{EtOH}$. RNA purity was assessed on a Nanodrop spectrometer. CDNA was synthesized using the iScript cDNA Synthesis Kit according to manufacturer's protocol (170-8891; Bio-Rad Laboratories). Quantitative PCR was performed with iQ SYBR ${ }^{\circledR}$ Green Supermix (170-8880; Bio-Rad Laboratories) according to the 
manufacturer's protocol on a QuantStudio 6 Flex Real-Time PCR System (Applied Biosystems), with data analyzed using GraphPad Prism 6 software. For the stem cell lineage plate array, RNA was collected using TRIzol as indicated above. CDNA was synthesized using the RT2 First Strand Synthesis Kit according to manufacturer's protocol (33041; Qiagen) qPCR primer sequences included:

$\begin{array}{ll}\text { Otx2_Forward } & \text { AATCAACTTGCCAGAATCCAGGG } \\ \text { Otx2_Reverse } & \text { GCTGTTGGCGGCACTTAGC } \\ \text { Cldn6_Forward } & \text { AGACAAAGCTGACCGAGCAC } \\ \text { Cldn6_Reverse } & \text { GTTGACCCAGCCAAGCAG } \\ \text { Rex1_Forward } & \text { CTCCTGCACACAGAAGAAAGC } \\ \text { Rex1_Reverse } & \text { CACTGATCCGCAAACACC } \\ \text { Stra8_Forward } & \text { GTTTCCTGCGTGTTCCACAAG } \\ \text { Stra8_Reverse } & \text { CACCCGAGGCTCAAGCTTC } \\ \text { Fgf5_Forward } & \text { CCTTGCGACCCAGGAGCTTA } \\ \text { Fgf5_Reverse } & \text { CCGTCTGTGGTTTCTGTTGAGG } \\ \text { Brachyury_Forward } & \text { CTGGGAGCTCAGTTCTTTCGA } \\ \text { Brachyury_Reverse } & \text { GAGGACGTGGCAGCTGAGA } \\ \text { TBP_Forward } & \text { GAAGAACAATCCAGACTAGCAGCA } \\ \text { TBP_Reverse } & \text { CCTTATAGGGAACTTCACATCACAG } \\ \end{array}$

\section{Immunoblotting}

Cells were lysed for 10 min in RIPA buffer (2.5 mM HEPES pH 7.5, $150 \mathrm{mM} \mathrm{NaCl}, 3 \mathrm{mM} \mathrm{KCl}$, $1 \%$ NP-40, $0.5 \%$ deoxycholate, $0.1 \%$ SDS, $1 \mathrm{mM}$ vanadate, and $5 \mathrm{mM} \mathrm{NaF}$ supplemented with protease and phosphatase inhibitors). Lysates were centrifuged at $13,000 \mathrm{rpm}$ for $15 \mathrm{~min}$ to obtain a post-nuclear supernatant. Proteins were separated by SDS-PAGE and transferred onto Immobilon-P® PVDF transfer membranes (IPVH00010; EMD Millipore) as previously described 
(Haynes et al., 2011; Rana et al., 2015). Membranes were blocked with 5\% non-fat milk in TBS containing $0.1 \%$ Tween (TBST) and incubated with primary antibodies overnight at $4^{\circ} \mathrm{C}$. Primary antibodies included a-tubulin (1:2000; GT114; GeneTex), Phospho-Myosin Light Chain 2 Thr18/Ser19 E2J8F (1:1000; \#95777; Cell Signaling Technology), ERK 1 C-16 (1:1000; sc-93; Santa Cruz Biotechnology, Inc.), Phospho-p44/42 MAPK Erk1/2 Thr202/Tyr204 (1:1000; \#9101; Cell Signaling Technology), Grhl2 (1:1000; HPA004820; Sigma), Arp2 (1:1000; A6104; Sigma), and Arpc2 (1:1000; 07-227; EMD Millipore). After washing, membranes were incubated in TBST with $5 \%$ non-fat milk and horseradish peroxidase (HRP)-conjugated secondary antibodies (1:10,000; 170-6516 and 172-1019; Bio Rad Laboratories) for $1 \mathrm{~h}$ at room temperature. After washing, immunoreactivity was developed with enhanced femto chemiluminescence (1859022 and 1859023; Thermo Scientific) and imaged using a BioRad Chemidoc XRS. ImageJ software was used for semi-quantitative densitometry analysis. Data presentation and statistical analysis were preformed using Excel Analyze-it and GraphPad Prism 6 software.

\section{Library preparation and RNA sequencing}

RNA was extracted with the RNeasy Mini kit (Qiagen, 74104) according to the manufacturer's instructions and sample concentrations were determined by NanoDrop. RNA degradation and contamination were monitored on $1 \%$ agarose gels, RNA purity was checked using the NanoPhotometer spectrophotometer (IMPLEN, CA, USA), and RNA integrity and quantitation were assessed using the RNA Nano 6000 Assay Kit of the Bioanalyzer 2100 system (Agilent Technologies, CA, USA). A total of 9 RNA libraries were prepared with three paired biological replicates for each condition including control +LIF2i, control 72h -LIF2i, and CK666 72h -LIF2i. A total amount of $1 \mu \mathrm{g}$ RNA per sample was used as input material for the RNA sample preparations. Sequencing libraries were generated using NEBNext Ultra RNA Library Prep Kit for Illumina (NEB, USA) following manufacturer's recommendations and index codes were added to attribute sequences to each sample. Briefly, mRNA was purified from total RNA using 
poly-T oligo-attached magnetic beads. Fragmentation was carried out using divalent cations under elevated temperature in NEBNext First Strand Synthesis Reaction Buffer (5X). First strand cDNA was synthesized using random hexamer primer and M-MuLV Reverse Transcriptase (RNase H). Second strand cDNA synthesis was subsequently performed using DNA Polymerase I and RNase $\mathrm{H}$. Remaining overhangs were converted into blunt ends via exonuclease/polymerase activities. After adenylation of 3' ends of DNA fragments, NEBNext Adaptor with hairpin loop structure was ligated to prepare for hybridization. To select cDNA fragments of preferentially 150 200 bp in length, the library fragments were purified with AMPure XP system (Beckman Coulter, Beverly, USA). Then $3 \mu$ USER Enzyme (NEB, USA) was used with size-selected, adaptor- ligated $\mathrm{cDNA}$ at $37^{\circ} \mathrm{C}$ for $15 \mathrm{~min}$ followed by 5 min at $95^{\circ} \mathrm{C}$ before PCR. Then PCR was performed with Phusion High-Fidelity DNA polymerase, Universal PCR primers and Index (X) Primer. At last, PCR products were purified (AMPure XP system) and library quality was assessed on the Agilent Bioanalyzer 2100 system. The clustering of the index-coded samples was performed on a cBot Cluster Generation System using PE Cluster Kit cBot-HS (Illumina) according to the manufacturer's instructions. After cluster generation, the library preparations were sequenced on an Illumina platform and pairedend reads were generated with $>20$ million reads per sample. The above protocol, with the exception of RNA extraction, was performed externally by Novogene Co. Ltd (USA).

\section{RNA sequencing analysis}

Quality assessment and basic processing of the reads was performed using the FastQC program (http://www.bioinformatics.babraham.ac.uk/projects/fastqc). Sequencing adapters were trimmed from the $3^{\prime}$ ends of the reads using cutadapt (v.1.8.1; https://pypi.python.org/pypi/cutadapt/1.8.1). We quantified transcript abundance with Kallisto (Bray et al., 2016) and built index with reference to the GRCh38 reference transcriptome. Expression analysis was performed using Sleuth (Pimentel et al., 2017) to 
assess differentially expressed genes between +LIF2i, control -LIF2i, and CK666 -LIF2i. Differentially expressed genes were identified using the Wald test with a cut-off of qval $<0.05$. Gene ontology enrichment analysis performed using Enrichr (Chen et al., 2013; Kuleshov et al., 2016). Heatmap figures were generated with Python using pandas dataframe (McKinney, 2010) input to the seaborn library (Waskom, 2021) in matplotlib (Hunter, 2007).

\section{Dataset acquisition}

CHIP-seq data of TBX3 binding in mESCs was available from NCBI (GEO Series accession number: GSE19219) (Han et al., 2010). Microarray data from shRNA TBX3 knockdown mESCs was available from NCBI (GEO Series accession number: GSE26520) (Nishiyama et al., 2013). RNA-seq data from TBX3-HI and TBX3-LO mESCs was available from NCBI (GEO Series accession number: GSE73862) (Russell et al., 2015). CHIP-seq data of MRTF binding in NIH3T3 fibroblasts was available from NCBI (GEO Series accession number: GSE45888) (Esnault et al., 2014).

\section{Data and code availability}

RNA-sequencing data generated during this study have been deposited in Gene Expression Omnibus (https://www.ncbi.nlm.nih.gov/geo/) under Accession code GEO: GSE175391. Software/packages used to analyze the dataset are freely available.

\section{Acknowledgments}

We thank A. Smith (University of Cambridge) for providing the E14 cell line and R. Blelloch (UCSF) for providing V6.5 wild type and DR cells. We also thank T. Nystul (UCSF), M. Welch (UC Berkeley), and the Barber Lab for helpful discussions. This work was supported by NIH grants GM116384 and CA197855 to DLB. FMA was supported by an HHMI Gilliam Fellowship, a UCSF Moritz-Heyman Discovery Fellowship, and an NIGMS T32GM008568 training grant. 


\section{Author contributions}

DLB conceived the hypothesis, which was developed by FMA. DLB and FMA obtained and analyzed data as well as assembled figures and wrote the manuscript.

The authors declare no competing interests.

\section{References}

Azami, T., Bassalert, C., Allègre, N., Estrella, L.V., Pouchin, P., Ema, M., and Chazaud, C. (2019). Regulation of the ERK signalling pathway in the developing mouse blastocyst.

Development 146(14), dev177139.

Baarlink, C., Wang, H., and Grosse, R. (2013). Nuclear actin network assembly by formins regulates the SRF coactivator MAL. Science 340(6134), 864-867.

Bailly, M., Ichetovin, I., Grant, W., Zebda, N., Machesky, L.M., Segall, J.E., and Condeelis, J. (2001). The F-actin side binding activity of the Arp2/3 complex is essential for actin nucleation and lamellipod extension. Curr Biol 11(8), 620-625.

Belin, B.J., and Mullins, R.D. (2013). What we talk about when we talk about nuclear actin. Nucleus 4(4), 291-297.

Bergert, M., Lembo, S., Sharma, S., Russo, L., Milovanović, D., Gretarsson, K.H., Börmel, M., Neveu, P.A., Hackett, J.A., Petsalaki, E., and Diz-Muñoz, A. (2021). Cell surfact mechanics gate embryonic stem cell differentiation. Cell Stem Cell 28, 209-216.

Betschinger, J., Nichols, J., Dietmann, S., Corrin, P.D., Paddison, P.J., and Smith, A. (2013). Exit from pluripotency is gated by intracellular redistribution of the bHLH transcription factor Tfe3. Cell 153(2), 335-347.

Bian, H., Lin, J.Z., Li, C., and Farmer, S.R. (2016). Myocardin-related transcription factor A (MRTFA) regulates the fate of bone marrow mesenchymal stem cells and its absence in mice leads to osteopenia. Mol Metab 5, 970-979.

Bongiorno, T., Gura, J., Talwar, P., Chambers, D., Young, K.M., Arafat, D., Wang, G., JacksonHolmes, E.L., Qiu, P., McDevitt., T.C., and Sulchek, T. (2018). Biophysical subsets of embryonic stem cells display distinct phenotypic and morphological signatures. PLOS ONE 13(3), e0192631.

Bray, N.L., Pimental, H., Melsted, P., and Pachter, L. (2016). Near-optimal probabilistic RNAseq quantification. Nat Biotechnol 34, 525-527. 
Brinkman, E.K., Chen, T., Amendola, M., and van Steensel, B. (2014). Easy quantitative assessment of genome editing by sequence trace decomposition. Nucleic Acids Res 42(22), e168.

Carlson, H., Ota, S., Campbell, C.E., and Hurlin, P.J. (2001). A dominant repression domain in Tbx3 mediates transcriptional repression and cell immortalization: relevance to mutations in Tbx3 that cause ulnar-mammary syndrome. Hum Mol Genet 10(21), 2403-2413.

Carroll, E.A., Gerrelli, D., Gasca, S., Berg, E., Beier, D.R., Copp, A.J., and Klingensmith, J. (2003). Cordon-bleu is a conserved gene involved in neural tube formation. Dev Biol 262(1), 1631.

Chapman, D.L., Garvey, N., Hancock, S., Alexiou, M., Agulnik, S.I., Gibson-Brown, J.J., CebraThomas, J., Bollag, R.J., Silver, L.M., and Papaioannou, V.E. (1996). Expression of the T-box family genes, Tbx1-Tbx5, during early mouse development. Dev Dyn 206(4), 379-390.

Charras, G., and Yap, A.S. (2018). Tensile Forces and Mechanotransduction at Cell-Cell Junctions. Curr Biol 28, R445-R457.

Chen, A.F., Liu, A.J., Krishnakumar, R., Freimer, J.W., DeVeale, B., and Blelloch, R. (2018). GRHL2-Dependent Enhancer Switching Maintains a Pluripotent Stem Cell Transcriptional Subnetwork after Exit from Naive Pluripotency. Cell Stem Cell 23(2), 226-238.

Chen, E.Y., Tan, C.M., Kou, Y., Duan, Q., Wang, Z., Meirelles, G.V., Clark, N.R., and Ma'ayan, A. (2013). Enrichr: interactive and collaborative HTML5 gene list enrichment analysis tool. BMC Bioinformatics 14, 128.

Chen, H., Guo, R., Zhang, Q., Guo, H., Yang, M., Wu, Z., Gao, S., Liu, L., and Chen, L. (2015). ERK signaling is indispensable for genomic stability and self-renewal of mouse embryonic stem cells. Proc Natl Acad Sci USA 112(44), E5936-43.

Chowdhury, F., Li, Y., Poh, Y.C., Yokohama-Tamaki, T., Wang, N., and Tanaka, T.S. (2010). Soft substrates promote homogenous self-renewal of embryonic stem cells via downregulating cell-matrix tractions. PLoS One 5(12), e15655.

Costello, I., Pimeisl, I.M., Dräger, S., Bikoff, E.K., Robertson, E.J., and Arnold, S.J. (2011). The T-box transcription factor Eomesodermin acts upstream of Mesp1 to specify cardiac mesoderm during mouse gastrulation. Nat Cell Biol 13(9), 1084-1091.

Di Nardo, A., Cicchetti, G., Falet, H., Hartwig, J.H., Stossel, T.P., and Kwiatkowski, D.J. (2005). Arp2/3 complex-deficient mouse fibroblasts are viable and have normal leading-edge actin structure and function. Proc Natl Acad Sci 102(45), 16263-16268.

De Belly, H., Stubb, A., Yanagida, A., Labouesse, C., Jones, P.H., Paluch, E.K., and Chalut, K.J. (2021). Membrane tension gates ERK-mediated regulation of pluripotent cell fate. Cell Stem Cell 28, 273-284.

Esnault, C., Stewart, A., Gualdrini, F., East, P., Horswell, S., Matthews, N., and Treisman, R. (2014). Rho-actin signaling to the MRTF coactivators dominants the immediate transcriptional response to serum in fibroblasts. Genes Dev 28(9), 943-958. 
Evans, M.J., and Kaufman, M.H. (1981). Establishment in culture of pluripotential cells from mouse embryos. Nature 292, 154-156.

Ganguly, A., Tang, Y., Wang, L., Ladt, K., Loi, J., Dargent, B., Leterrier, C., and Roy, S. (2015). A dynamic formin-dependent deep F-actin network in axons. J Cell Biol 210(3), 401-417.

Gilmour, D., Rembold, M., and Leptin, M. (2017). From morphogen to morphogenesis and back. Nature 541(7637), 311-320.

Goley, E.D., and Welch, M.D. (2006). The Arp2/3 complex: an actin nucleator comes of age. Nat Rev Mol Cell Biol 7(10), 713-726.

Hamilton, W.B., Kaji, K., and Kunath, T. (2013). ERK2 suppresses self-renewal capacity of embryonic stem cells, but is not required for multi-lineage commitment. PLoS One 8(4), e60907.

Han, J., Yuan, P., Yang, H., Zhang, J., Soh, B.S., Li, P., Lim, S.L., Cao, S., Tay, J., Orlov, Y.L., et al. (2010). Tbx3 improves the germ-line competency of induced pluripotent stem cells. Nature 463(7284), 1096-1100.

Happe, C.L., and Engler, A.J. (2016). Mechanical Forces Reshape Differentiation Cues That Guide Cardiomyogenesis. Circ Res 118(2), 296-310.

Hunter, J.D. (2007). Matplotlib: A 2D graphics environment. Comput Sci Eng 9(3), 90-95.

Hurst, V., Shimada, K., and Gasser, S.M. (2019). Nuclear Actin and Actin-Binding Proteins in DNA Repair. Trends Cell Biol 29(6), 462-476.

Iskratsch, T., Wolfenson, H., and Sheetz, M.P. (2014). Appreciating force and shape - the rise of mechanotransduction in cell biology. Nat Rev Mol Cell Biol 15, 825-833.

Janmey, P.A., Wells, R.G., Assoian, R.K., and McCulloch, C.A. (2013). From tissue mechanics to transcription factors. Differentiation 86(3), 112-120.

Jurmeister, S., Baumann, M., Balwierz, A., Keklikoglou, I., Ward, A., Uhlmann, S., Zhang, J.D., Wiemann, S., and Sahin, O. (2012). MicroRNA-200c represses migration and invasion of breast cancer cells by targeting actin-regulatory proteins FHOD1 and PPM1F. Mol Cel Biol 32, 633651.

Kalkan, T., and Smith, A. (2014). Mapping the route from naive pluripotency to lineage specification. Philos Trans R Soc Lond B Biol Sci 369, 20130540.

Kalkan, T., Olova, N., Roode, M., Mulas, C., Lee, H.J., Nett, I., Marks, H., Walker, R., Stunnenberg, H.G., Lilley, K.S., et al. (2017). Tracking the embryonic stem cell transition from ground state pluripotency. Development 144, 1221-1234.

Kalkan, T., Bornelöv, S., Mulas, C., Diamanti, E., Lohoff, T., Ralser, M., Middelkamp, S., Lombard, P., Nichols, J., and Smith, A. (2019). Complementary Activity of ETV5, RBPJ, and TCF3 Drives Formative Transition from Naïve Pluripotency. Cell Stem Cell 24(5), 785-801.

Kartikasari, A.E.R., Zhou, J.X., Kanji, M.S., Chan, D.N., Sinha, A., Grapin-Botton, A., Magnuson, M.A., Lowry, W.E., and Bhusan, A. (2013). EMBO J 32(10), 1393-1408. 
Kerosuo, L., Piltti, K., Fox, H., Angers-Loustau, A., Häyry, V., Eilers, M., Sariola, H., and Wartiovaara, K. (2008). Myc increases self-renewal in neural progenitor cells through Miz-1. J Cell Sci 121(23), 3941-3950.

Kinoshita, M., and Smith, A. (2018). Pluripotency Deconstructed. Dev Growth Differ 60(1), 4452.

Keller, R., Davidson, L.A., and Shook, D.R. (2003). How we are shaped: The biomechanics of gastrulation. Differentiation 71, 171-205.

Knoepfler, P.S., Cheng, P.F., and Eisenman, R.N. (2002). N-myc is essential during neurogenesis for the rapid expansion of progenitor cell populations and the inhibition of neuronal differentiation. Genes Dev 16(20), 2699-2712.

Krieg, M., Arboleda-Estudillo, Y., Puech, P.H., Käfer, J., Graner, F., Müller, D.J., and Heisenberg, C.P. (2008). Tensile forces govern germ-later organization in zebrafish. Nat Cell Biol 10(4), 429-436.

Kuleshov, M.V., Jones, M.R., Rouillard, A.D., Fernandez, N.F., Duan, Q., Wang, Z., Koplev, S., Jenkins, S.L., Jagodnik, K.M., Lachmann, A., et al. (2016). Enrichr: a comprehensive gene set enrichment analysis web server 2016 update. Nucleic Acids Res 44(W1), W90-W97.

Le, H.Q., Ghatak, S., Yeung, C.Y., Tellkamp, F., Günschmann, C., Dieterich, C., Yeroslaviz, A., Habermann, B., Pombo, A., Niessen, C.M., and Wickström, S.A. (2016). Nat Cell Biol 18(8), 864-875.

LeClaire, L.L., Rana, M., Baumgartner, M., and Barber, D.L. (2015). The Nck-interacting kinase NIK increases Arp2/3 complex activity by phosphorylating the Arp2 subunit. J Cell Biol 208(2), 161-170.

Lee, J., Abdeen, A.A., Zhang, D., and Kilian, K.A. (2013). Directing stem cell fatr on hydrogel substrates by controlling cell geometry, matrix mechanics and adhesion ligand composition. Biomaterials 34(33), 8140-8148.

Li, Y., Zhu, X., Zeng, Y., Wang, J., Zhang, X., Ding, Y.Q., and Liang, L. (2010). FMNL2 enhances invasion of colorectal carcinoma by inducing epithelial-mesenchymal transition. Mol Cancer Res 8, 1579-1590.

Lorda-Diez, C.I., Montero, J.A., Sanchez-Fernandez, C., Garcia-Porrero, J.A., Chimal-Monroy, J., and Hurle, J.M. (2018). Four and a half domain 2 (FHL2) scaffolding protein is a marker of connective tissues of developing digits and regulates fibrogenic differentiation of lim mesodermal progenitors. J Tissue Eng Regen Med 12(4), e2062-e2072.

Lu, R., Yang, A., and Jin, Y. (2011). Dual functions of T-box 3 (Tbx3) in the control of selfrenewal and extraembryonic endoderm differentiation in mouse embryonic stem cells. J Biol Chem 286(10), 8425-8436.

Martin, G.R. (1981). Isolation of a pluripotent cell line from early mouse embryos cultured in medium conditioned by teratocarcinoma stem cells. Proc. Natl. Acad. Sci. 78(12), 7634-7638. 
May, R. (2001). The Arp2/3 complex: a central regulator of the actin cytoskeleton. Cell Mol Life Sci 58(11), 1607-1626.

McDonald, M.E., Li, C., Bian, H., Smith, B.D., Layne, M.D., and Farmer, S.R. (2015).

Myocardin-related transcription factor $A$ regulates conversion of progenitors to beige adipocytes. Cell 160, 105-118.

McGee, K.M., Vartiainen, M.K., Khaw, P.T., Treisman, R., and Bailly, M. (2011). Nuclear transport of the serum response factor coactivator MRTF-A is downregulated at tensional homeostasis. EMBO Rep 12(9), 963-970.

McKinney, W. (2010). Data structures for statistical computing in python. In S. van der Walt \& J. Millman (Eds.), Proceedings of the $9^{\text {th }}$ Python in Science Conference, 51-56.

Miralles, F., Posern, G., Zaramytidou, A.I., and Treisman, R. (2003). Actin dynamics control SRF activity by regulation of its coactivator MAL. Cell 113(3), 329-342.

Molé, M.A., Weberling, A., Fässler, R., Campbell, A., Fishel, S., and Zernicka-Goetz, M. (2021). Integrin $B 1$ coordinates survival and morphogenesis of the embryonic lineage upon implantation and pluripotency transition. Cell Rep 34, 108834.

Mulas, C., Kalkan, T., and Smith, A. (2017). NODAL Secures Pluripotency upon Embryonic Stem Cell Progression from the Ground State. Stem Cell Rep 9(1), 77-91.

Murrell, M., Oakes, P.W., Lenz, M., and Gardel, M.L. (2015). Forcing cells into shape: the mechanics of actomyosin contractility. Nat Rev Mol Cell Biol 16, 486-498.

Nakazawa, N., Sathe, A.R., Shivashankar, G.V., and Sheetz, M.P. (2016). Matrix mechanics controls FHL2 movement to the nucleus to activate p21 expression. Proc Natl Acad Sci USA 113(44), E6813-E6822.

Närvä, E., Stubb, A., Guzmán, C., Blomqvist, M., Balboa, D., Lerche, M., Saari, M., Otonkoski, T., and Ivaska, J. (2017). A strong contractile actin fence and large adhesions direct human pluripotent colony morphology and adhesion. Stem Cell Rep 9(1), 67-76.

Nichols, J., and Smith, A. (2009). Naive and primed pluripotent states. Cell Stem Cell 4(6), 487492.

Nichols, J., and Smith, A. (2012). Pluripotency in the embryo and in culture. Cold Spring Harb Perspect Biol 4(8): a008128.

Nishimura, Y., Shi, S., Zhang, F., Liu, R., Takagi, Y., Bershadsky, A.D., Viasnoff, V., and Sellers, J.R. (2021). The formin inhibitor, SMIFH2, inhibits members of the myosin superfamily. J Cell Sci doi: 10.1242/jcs.253708, Online ahead of print.

Nishiyama, A., Sharov, A.A., Piao, Y., Amano, M., Amano, T., Hoang, H.G., Binder, B.Y., Tapnio, R., Bassey, U., Malinou, J.N., et al. (2013). Systematic repression of transcription factors reveals limited patterns of gene expression changes in ES cells. Sci Rep 3(1390), srep01390. 
Niwa, H., Ogawa, K., Shimosato, D., and Adachi, K. (2009). A parallel circuit of LIF signalling pathways maintains pluripotency of mouse ES cells. Nature 460(7251), 118-122.

Nobusue, H., Onishi, N., Shimizu, T., Sugihara, E., Oki, Y., Sumikawa, Y., Chiyoda, T., Akashi, K., Saya, H., and Hano, K. (2014). Reglation of MKL1 via actin cytoskeleton dynamics drives adipocyte differentiation. Nat Commun 5, 3368.

Nolen, B.J., Tomasevic, N., Russell, A., Pierce, D.W., Jia, Z., McCormick, C.D., Hartman, J., Sakowicz, R., and Pollard, T.D. (2009). Nature 460(7258), 1031-1034.

Ohinata, Y., Payer, B., O’Carroll, D., Ancelin, K., Ono, Y., Sano, M., Barton, S.C., Obukhanych, T., Nussenzweig, M., Tarakhovsky, A., et al. (2005). Blimp1 is a critical determinant of germ cell lineage in mice. Natura 436(7048), 207-213.

Olson, E.M., and Nordheim, A. (2010). Linking actin dynamics and gene transcription to drive cellular motile functions. Nat Rev Mol Cell Biol 11(5), 353-365.

Parchem, R.J., Ye, J., Judson, R.L., LaRussa, M.F., Krishnakumar, R., Blelloch, A., Oldham, M.C., and Blelloch, R. (2014). Two miRNA clusters reveal alternative paths in late-stage programming. Cell Stem Cell 14(5), 617-631.

Philippar, U., Schratt, G., Dieterich, C., Müller, J.M., Galgóczy, P., Engel, F.B., Keating, M.T., Gertler, F., Schüle, R., Vingron, M., and Nordheim, A. (2004). The SRF target gene Fhl2 antagonizes RhoA/MAL-depednet activation of SRF. Mol Cell 16(6), 867-880.

Pieters, T. and van Roy, F. (2014). Role of cell-cell adhesion complexes in embryonic stem cell biology. J Cell Sci 127(12), 2603-2613.

Pimental, H., Bray, N.L., Puente, S., Melsted, P., and Pachter, L. (20). Differential analysis of RNA-seq incorporating quantification uncertainty. Nat Methods 14(7), 687-690.

Posern, G., and Treisman, R. (2006). Actin' together: serum response factor, its cofactors and the link to signal transduction. Trends Cell Biol 16(11), 588-596.

Prajapati, R.S., Hintze, M., and Streit, A. (2019). PRDM1 controls the sequential activation of neural, neural crest and sensory progenitor determinants. Development 146(24), dev181107.

Ran, F.A., Hsu, P.D., Wright, J., Agarwala, V., Scott, D.A., and Zhang, F. (2013). Genome engineering using the CRISPR-Cas9 system. Nat Protoc 8(11), 2281-2308.

Rana, M.K., Aloisio, F.M., Choi, C., and Barber, D.L. (2018). Formin-dependent TGF- $\beta$ signaling for epithelial to mesenchymal transition. Mol Biol Cell 29(12), 1465-1475.

Rauhala, H.E., Teppo, S., Niemelä, S., and Kallioniemi, A. (2013). Silencing of the ARP2/3 complex disturbs pancreatic cancer cell migration. Anticancer Res 33(10), 45-52.

Renger, A., Zafiriou, M., Noack, C., Pavlova, E., Becker, A., Sharkova, K., Bergmann, M.W., ElArmouche, A., Zimmerman, E., and Zelarayán, L.C. (2013). The Four and a Half LIM-domain 2 controls early crdiac cell commitment and expansion via regulating ß-catenin-dependent transcription. Stem Cells 31(5), 928-940. 
Rizvi, S.A., Neidt, E.M., Cui, J., Feiger, Z., Skau, C.T., Gardel, M.L., Kozmin, S.A., and Kovar, D.R. (2009). Identification and characterization of a small molecule inhibitor of formin-mediated actin assembly. Chem Biol 16(11), 1158-1168.

Rotty, J.D., Wu, C., and Bear, J.E. (2013) New insights into the regulation and cellular functions of the ARP2/3 complex. Nat Rev Mol Cell Biol 14(1), 7-12.

Russell, R., Ilg, M., Lin, Q., Wu, G., Lechel, A., Bergmann, W., Eiseler, T., Linta, L., Kumar, P., Klingenstein, M., et al. (2015). A dynamic role of Tbx3 in the pluripotency circuitry. Stem Cell Rep 5(6), 1155-1170.

Sanjana, N.E., Shalem, O., and Zhang, F. (2014). Improved vectors and genome-wide libraries for CRISPR screening. Nat Methods 11(8), 783-784.

Schratt, G., Phillippar, U., Berger, J., Schwarz, H., Heidenreich, O., and Nordheim, A. (2002). Serum response factor is crucuail for actin cytoskeletal organization and focal adhesion assembly in embryonic stem cells. J Cell Biol 156(4), 737-750.

Sen, B., Xie, Z., Uzer, G., Thompson, W.R., Styner, M., Wu, X., and Rubin, J. (2015). Intranuclear Actin Regulates Osteogenesis. Stem Cells 33(10), 3065-3076.

Smith, A. (2017). Formative pluripotency: the executive phase in a developmental continuum. Development 144, 365-373.

Steffen, A., Faix, J., Resch, G.P., Linkner, J., Wehland, J., Small, J.V., Rottner, K., and Stradal, T.E. (2006) Filopodia formation in the absence of functional WAVE- and Arp2/3-complexes. Mol Biol Cell 17(6), 2581-2591.

Sun, Q., Chen, G., Streb, J.W., Long, X., Yang, Y., Stoeckart, C.J. Jr., and Milano, J.M. (2006). Defining the mammalian CArGome. Genome Res 16(2), 197-207.

Sun, X., Phua, D.Y.Z., Axiotakis, Jr., L., Smith, M.A., Blankman, E., Gong, R., Cail, R.C., Espinosa de los Reyes, S., Beckerle, M.C., Waterman, C.M., and Alushin, G.M. (2020). Mechanosensing through direct binding of tensed F-actin by LIM domains. Dev Cell 55, 1-15.

Suraneni, P., Rubinstein, B., Unruh, J.R., Durnin, M., Hanein, D., and Li, R. (2012). The Arp2/3 complex is required for lamellipodia extension and directional fibroblast cell migration. J Cell Biol 197(2), 239-251.

Swaney, K.F., and Li, R. (2016). Function and regulation of the Arp2/3 complex during cell migration in diverse environments. Curr Opin Cell Biol 42, 63-72.

Tatapudy, S., Aloisio, F., Barber, D., and Nystul, T. (2017). Cell fate decisions: emerging roles for metabolic signals and cell morphology. EMBO Rep 18(20), 2105-2118.

Tosic, J., Kim, G., Pavlovic, M., Schröder, C.M., Mersiowsky, S, Barg, M., Hofherr, A., Probst, S., Köttgen, M., Hein, L., and Arnold, S.J. (2019). Eomes and Brachyury control pluripotency exit and germ-layer segregation by changing the chromatin state. Nat Cell Biol 21, 1518-1531. 
Vartiainen, M.K., Guettler, S., Larijani, B., and Treisman, R. (2007). Nuclear actin regulates dynamic subcellular localization and activity of the SRF cofactor MAL. Science 316(5832), 1749-1752.

Verma, S., Han, S.P., Michael, M., Gomez, G.A., Yang, Z., Teasdale, R.D., Ratheesh, A., Kovacs, E.M., Ali, R.G., and Yap, A.S. (2012). A WAVE2-Arp2/3 actin nucleator apparatus supports junctional tension at the epithelial zonula adherens. Mol Biol Cel 23(23), 4601-4610.

Villegas, F., Lehalle, D., Mayer, D., Rittirsch, M., Stadler, M.B., Zinner, M., Olivieri, D., Vabres, P., Duplomb-Jego, L., De Bont, E.S.J.M., et al. (2019). Lysosomal Signaling Licenses Embryonic Stem Cell Differentiation via Inactivation of Tfe3. Cell Stem Cell 24(2), 257-270.

Villeneuve, C., Wickström, S.A. (2021). Shaping the stem cell field. Nat Rev Mol Cell Biol 22, 305.

Wagh, K., Ishikawa, M., Garcia, D.A., Stavreva, D.A., Upadhyaya, A., and Hager, G.L. (2021). Mechanical regulation of transcription: Recent Advances. Trends Cell Biol 31(6), 457-472.

Wang, D.Z., Li, S., Hockemeyer, D., Sutherland, L., Wang, Z., Schratt, G., Richardson, J.A., Nordheim, A., and Olson, E.N. (2002). Potentiation of serum response factor activity by a family of myocardin-related transcription factors. Proc Natl Acad Sci USA 99(23), 14855-14860.

Waskom, M.L. (2021). Seaborn: statistical data visualization. J Open Source Softw 6(60), 3021.

Weidgang, C.E., Russell, R., Tata, P.R., Kühl, S.J., Illing, A., Müller, M., Lin, Q., Brunner, C., Boeckers, T.M., Bauer, K., et al. (2013). TBX3 directs cell-fate decision toward mesendoderm. Stem Cell Rep 1(3), 248-265.

Weinberger, L., Ayyash, M., Novershtern, N., and Hanna, J.H. (2016). Dynamic stem cell states: naive to primed pluripotency in rodents and humans. Nat Rev Mol Cell Biol 17(3), 155-169.

Weinhold, B., Schratt, G., Arsenian, S., Berger, J., Kamino, K., Schwarz, H., Rüther, U., and Nordeim, A. (2000). Srf-/- ES cells display non-cell-autonomous impairment in mesodermal differentiation. EMBO J 19(21), 5835-5844.

Xia, S., Lim, Y.B., Zhang, Z., Wang, Y., Zhang, S., Lim, C.T., Yim, E.K.F., Kanchanawong, P. (2019). Nanoscale architecture of the cortical actin cytoskeleton in embryonic stem cells. Cell Rep 28, 1251-1267.

Yang, Q., Zhang, X.F., Pollard, T.D., and Forscher, P. (2012). Arp2/3 complex-dependent actin networks constrain myosin II function in driving retrograde actin flow. J Cell Biol 197(7), 939956.

Ying, Q.L., Wray, J., Nichols, J., Batlle-Morera, L., Doble, B., Woodgett, J., Cohen, P., and Smith, A. (2008). The ground state of embryonic stem cell self-renewal. Nature 453(7194), 519523.

Yoo, Y., Wu, X., and Guan, J.L. (2007). A novel role of the actin nucleating Arp2/3 complex in the regulation of RNA polymerase II-dependent transcription. J Biol Chem 282(10), 7616-7623. 
You, Y., Ramachandra, S.G., and Jin, T. A CRISPR-based method for testing the essentiality of a gene. Sci Rep 10(1), 14779.

\section{Figure Legends}

Figure 1 Inhibiting Arp2/3 complex but not formin activity blocks morphological changes and actin remodeling during mESC differentiation

(A) DIC images of E14 mESCs over 72h -LIF2i in the absence or presence of CK666 or

SMIFH2. (B) Colony circularity quantified from DIC images in (A). Box plots show median, first and third quartile, with whiskers extending to observations within 1.5 times the interquartile range, and all individual data points representing means obtained from 6 independent cell preparations of 15-20 individual colonies each (C) Confocal images of E14 mESCs +LIF2i and LIF2i for $72 \mathrm{~h}$ in the absence or presence of CK666 or SMIFH2 and with Arpc2 KD labeled for Factin with rhodamine phalloidin. (D) Confocal images of V6.5 mESCs +LIF2i and -LIF2i for $72 \mathrm{~h}$ in the absence or presence of $\mathrm{CK} 666$ or SMIFH2 labeled for F-actin with rhodamine phalloidin. (E) Confocal images of E14 mESCs +LIF2i and at 72h -LIF2i in the absence or presence of CK666 or SMIFH2 and immunolabeled for pMLC (green) and stained for F-actin with rhodamine phalloidin (magenta) or nuclei with DAPI (blue). Data were analyzed by two-tailed unpaired Student's $t$-test with a significance level of $p<0.05$.

Figure 2 Inhibiting Arp2/3 complex but not formin activity impairs differentiation to EpiLCs

(A) Schematic of DR mESCs indicating naive self-renewing mESCs expressing miR-290mCherry, primed EpiLCs expressing miR-302-eGFP, and cells transitioning between these stages expressing both markers. (B) FACS of V6.5 DR mESCs after 72h -LIF2i in the absence or presence of CK666, CK689 inactive analog of CK666, SMIFH2 or DMSO vehicle, with indicated data representing a mean from 6 independent cell preparations. (C-E) RT-qPCR for Fgf5 (C) and Brachyury (D) in V6.5 DR mESCs and for Fgf5 in E14 mESCs (E) +LIF2i and at 
72h -LIF2i. Conditions include the absence (controls) or presence of CK666 or CK689 (C,D) and control and ARPC2 KD cells (E), with data showing the means \pm SEM of 3 independent cell preparations normalized to TBP. (F) Confocal images of E14 mESCs +LIF2i and at 72h -LIF2i in the absence or presence of CK666 and immunolabeled for TFE3 (green) and stained for F-actin with rhodamine phalloidin (magenta). (G) Quantified nuclear to cytoplasmic ratio of TFE3 immunolabeling shown in $(F)$ indicating means \pm SEM of 3 independent cell preparations. Data were analyzed by two-tailed unpaired Student's $t$-test with a significance level of $p<0.05$.

Figure 3 Inhibiting Arp2/3 complex activity has no effect on exit from naive self-renewal but delays entry into formative pluripotency

(A,B) RT-qPCR for Rex1 in V6.5 DR mESCs (A) and E14 mESCs (B) +LIF2i and at 72h -LiF2i in untreated control cells and in the presence of CK666 or the inactive CK666 analog CK689 (A) or in ARPC2 KD cells (B). Data are means \pm SEM of 3 independent cell preparations normalized to TBP. (C) RT-qPCR for Rex1 in E14 mESCs during 120h time-course -LIF2i in untreated control cells and in the presence of CK666. Data are means \pm SEM of 4 independent cell preparations normalized to TBP. (D) RT-qPCR for Otx2 in E14 mESCs during 48h timecourse -LIF2i in untreated control cells and in the presence of CK666. Data are means \pm SEM of 4 independent cell preparations normalized to TBP. (E) Representative immunoblot of lysates from E14 mESCs during 48h time-course -LIF2i in untreated control cells and in the presence of CK666 probed for pERK, total ERK, or tubulin as a loading control. (F) Semiquantitative densitometry of immunoblots described in $(E)$, with data showing means \pm SEM of 3 independent cell preparations. (G) Representative immunoblot of lysates from E14 mESCs during 48h time-course -LIF2i in untreated control cells and in the presence of CK666 probed for GRHL2 or tubulin as a loading control. (H) Semiquantitative densitometry of immunoblots described in $(G)$, with data showing means \pm SEM of 7 independent cell preparations. (I) RT- 
qPCR for Cldn6 in E14 mESCs after 24h -LIF2i in untreated control cells and in the presence of CK666. Data are means \pm SEM of 5 independent cell preparations normalized to TBP. Given directional a priori predictions in panels $\mathrm{D}-\mathrm{H}$, data were analyzed by one-tailed unpaired Student's $t$-test with a significance level of $p<0.05$.

Figure 4 Inhibiting Arp2/3 complex activity causes global defects in lineage specification

(A) Volcano plot showing the transcriptome fold-changes (beta values) in Control -LIF2i compared with +LIF2i E14 mESCs after 72h. Each dot represents one gene with significantly changed genes (q-value $<0.05)$ indicated in blue and significantly changed genes of interest indicated in red. (B) Volcano plot showing the transcriptome fold-changes (beta values) in CK666 -LIF2i compared with Control -LIF2i E14 mESCs after 72h. Each dot represents one gene with significantly changed genes (q-value $<0.05)$ indicated in blue and significantly changed genes of interest indicated in red. (C) Venn diagram showing the number of shared and distinct DEGs indicated by RNA-seq for each listed comparison. (D) GO Biological Process (2019) enrichment analysis of 972 DEGs uniquely indicated in CK666 -LIF2i compared to Control -LIF2i after 72h. (E-H) Clustermap showing naive mESC marker (E), mesoderm marker (F), endoderm marker (G), and ectoderm marker $(\mathbf{H})$ expression indicated by beta values from RNA-seq analysis of E14 mESCs in the absence (Control) or presence of CK666 after 72h LIF2i compared to +LIF2i.

Figure 5 Inhibiting Arp2/3 complex activity disrupts Tbx3-dependent transcriptional programs

(A-C) Clustermap showing expression of Tbx3 target genes identified by Russell et al., 2015 (A), Nishiyama et al., 2013 (B), or Han et al., 2010 (C) with beta value fold-changes indicated from RNA-seq analysis of E14 mESCs in a control differentiation (Control -LIF2i vs +LIF2i) and how they are affected in the presence of CK666 (CK666 -LIF2i vs Control -LIF2i). (D) Enriched 
bar graph with beta value fold-changes indicated from RNA-seq analysis of E14 mESCs for commonly identified TBX3 target genes across all three published datasets (Russell et al., 2015; Nishiyama et al., 2013; Han et al., 2010) which exhibit significantly different expression (qval < 0.05) in CK666 -LIF2i compared to control -LIF2i.

Figure 6 Inhibiting Arp2/3 complex activity blocks cytoplasmic and nuclear shuttling of FHL2 and MRTF

(A) Confocal images of E14 mESCs +LIF2i and at 72h -LIF2i in the absence or presence of CK666 or SMIFH2 immunolabeled for MRTF (green) and stained for F-actin with rhodamine phalloidin (magenta) and for nuclei with DAPI (blue). (B) Quantified nuclear to cytoplasmic ratio of MRTF immunolabeling shown in $(A)$ indicating means \pm SEM of 3 independent cell preparations. (C) Clustermap showing expression of MRTF target genes identified by Esnault et al., 2014 with beta values indicated from RNA-seq analysis of E14 mESCs in a control differentiation (Control -LIF2i vs +LIF2i) and how they are affected in the presence of CK666 (CK666 -LIF2i vs Control -LIF2i). (D) Confocal images of E14 mESCs +LIF2i and at 72h -LIF2i in the absence or presence of CK666 immunolabeled for FHL2 (green) and stained for F-actin with rhodamine phalloidin (magenta) and for nuclei with DAPI (blue). (E) Quantified nuclear to cytoplasmic ratio of FHL2 immunolabeling shown in (D) indicating means \pm SEM of cells from images obtained in 4 independent cell preparations. Data were analyzed by two-tailed unpaired Student's $t$-test with a significance level of $p<0.05$. (F) Model of competing inverse actin-dependent MRTF/FHL2 nuclear translocation for mESCs in the presence and absence of LIF2i. Created with BioRender.com.

\section{Supplemental Video 1}


E14 colony morphology in the presence of LIF2i. DIC time-lapse recording of E14 cells with images taken every 10 min for $18 \mathrm{~h}$ on gelatin-coated glass, $40 \mathrm{x}$.

\section{Supplemental Video 2}

E14 colony morphology at $\mathbf{2 4 - 4 2 h}$-LIF2i. DIC time-lapse recording of E14 cells with images taken every $10 \mathrm{~min}$ for $18 \mathrm{~h}$ on gelatin-coated glass, $40 \mathrm{x}$.

\section{Supplemental Figure 1}

(A) Representative immunoblot of lysates from E14 control or Arpc2 KD mESCs probed for ARPC2 or tubulin as a loading control. (B) Semiquantitative densitometry of immunoblots described in $(A)$, with data showing means \pm SEM of 3 independent cell preparations. (C) Representative immunoblot of lysates from E14 control or Arpc2 KD mESCs probed for ARP2 or tubulin as a loading control. (D) Semiquantitative densitometry of immunoblots described in (C), with data showing means \pm SEM of 3 independent cell preparations. (E) Profile of CRISPRCas9 edited E14 mESCs using TIDE webtool (https://tide.deskgen.com) to quantify editing efficacy by sequence aberration compared to control cells and to (F) identify the predominant indel (Brinkman et al., Nucleic Acids Reserch 2014). (G) Representative immunoblot of lysates from E14 mESCs over 72h timecourse -LIF2i in the absence (control) or presence of CK666 or SMIFH2 probed for pMLC and tubulin as a loading control. (H) Semiquantitative densitometry of immunoblots described in $(G)$, with data showing means \pm SEM of 3 independent cell preparations. Data were analyzed by two-tailed unpaired Student's $t$-test with a significance level of $p<0.05$.

\section{Supplemental Figure 2}

(A) FACS of V6.5 DR mESCs at 72h with LIF2i in the absence (control) or presence of CK666, CK689 inactive analog of CK666, SMIFH2, or DMSO vehicle, with indicated data from 6 
independent cell preparations. (B) FACS of V6. 5 DR mESCs stained with DAPI during $72 \mathrm{~h}$ LIF2i timecourse in the absence (control) or presence of CK666, CK689 inactive analog of CK666, SMIFH2, or DMSO vehicle, with indicated data from 6 independent cell preparations to identify the differentiation status of only dead or dying cells. (C) RT-qPCR for Rex1 and (D) Stra8 in E14 mESCs at 120h with LIF2i in the absence (control) or presence of CK666 with indicated data showing means \pm SEM of 4 independent cell preparations normalized to TBP. (E) Number of E14 mESCs after 72h -LIF2i in the absence (control) or presence of CK666, CK689 inactive analog of CK666, SMIFH2, or DMSO vehicle, with indicated data showing means \pm SEM of 3 independent cell preparations. (F) RT-qPCR for Stra8 in E14 mESCs during 120h LIF2i timecourse in the absence (control) or presence of CK666, with indicated data showing means \pm SEM of 4 independent cell preparations normalized to TBP. Data were analyzed by two-tailed unpaired Student's $t$-test with a significance level of $p<0.05$.

\section{Supplemental Figure 3}

(A) Confocal images of E14 mESCs +LIF2i and at 120h -LIF2i in the absence or presence of CK666 immunolabeled for MRTF (green) and stained for F-actin with rhodamine phalloidin (magenta) and for nuclei with DAPI (blue). (B) Quantified nuclear to cytoplasmic ratio of MRTF immunolabeling shown in $(A)$ indicating means \pm SEM of 3 independent cell preparations. Data were analyzed by two-tailed unpaired Student's $t$-test with a significance level of $p<0.05$. 
bioRxiv preprint doi: https://doi.org/10.1101/2021.11.02.465785; this version posted November 4, 2021. The copyright holder for this preprint (which was not certified by peer review) is the author/funder, who has granted bioRxiv a license to display the preprint in perpetuity. It is made available under aCC-BY-NC-ND 4.0 International license. 


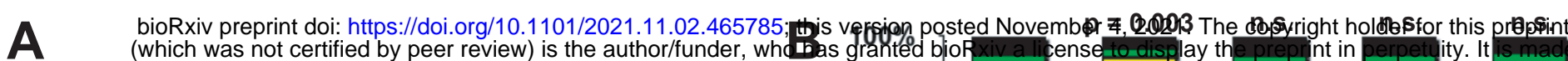

Naive ESC mCherry (+)

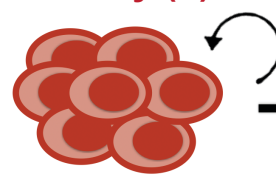

miR-290

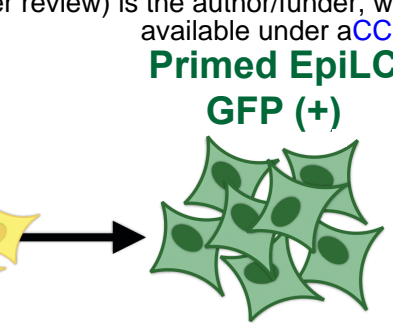

miR-302
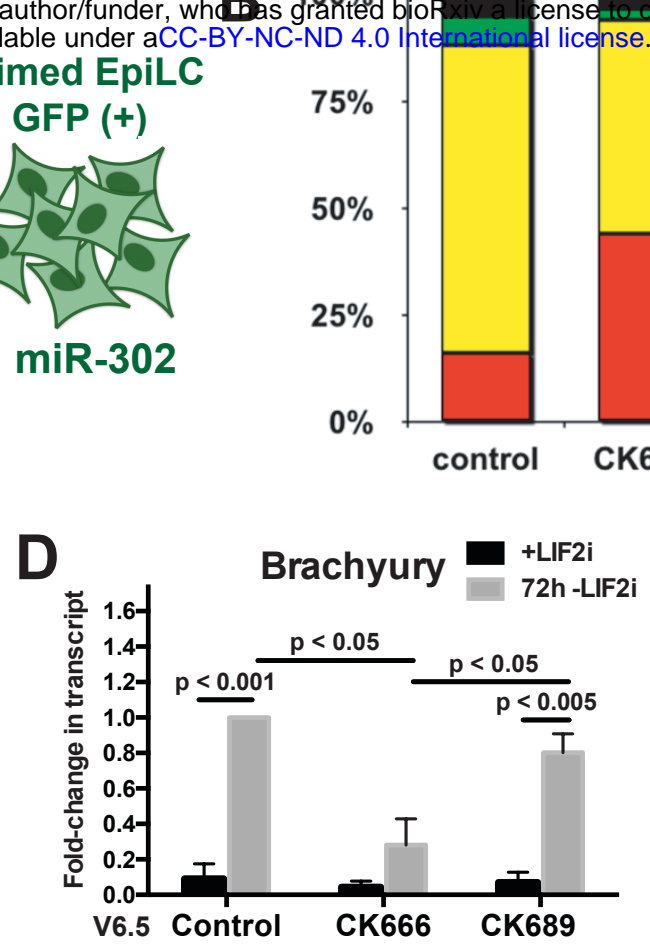

E

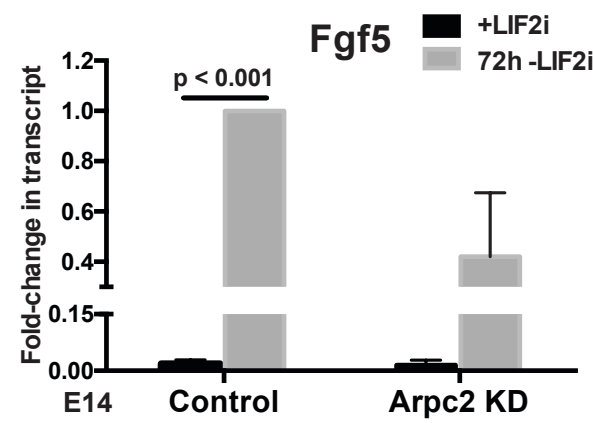

F

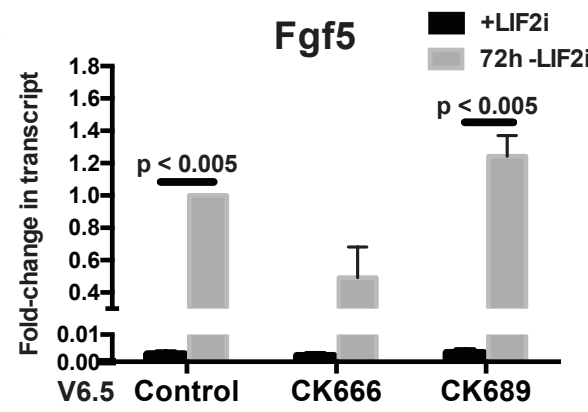

F

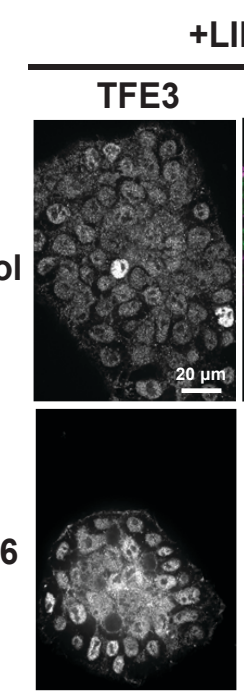

+LIF2i
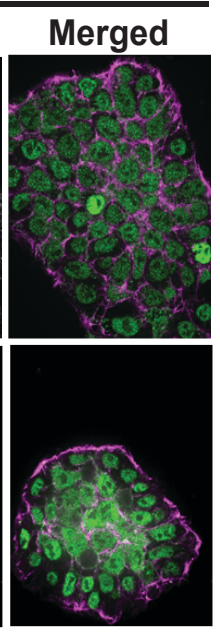

-LIF2i

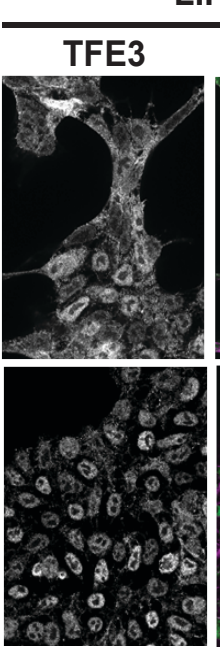

G

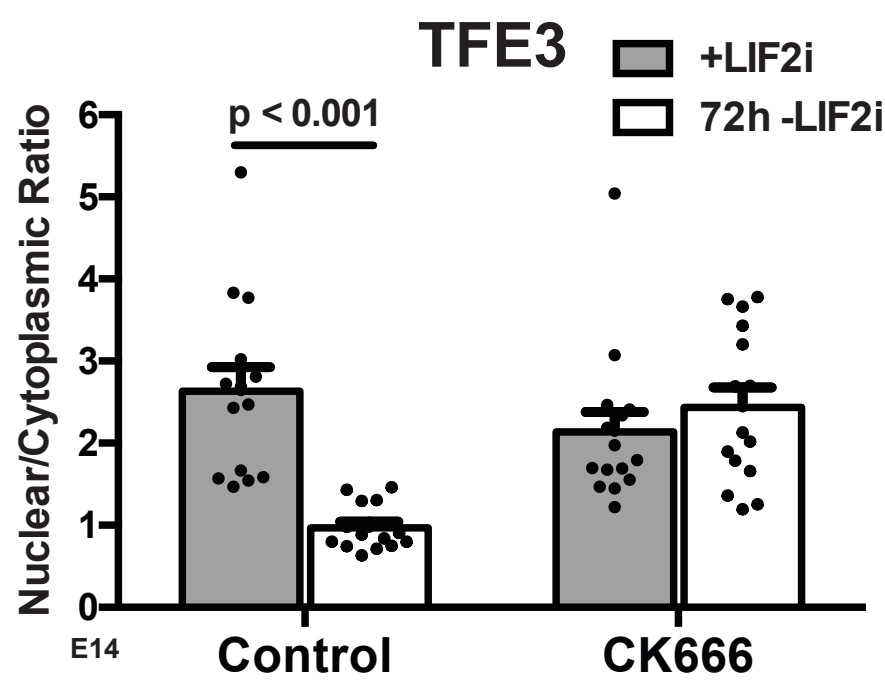


bioRxiv preprint doi: https://doi.org/10.1101/PQ21.11.02.465785; this version posted November 4,2981 . The copyright holder for this preprint

A (which was not certified by peer review) is th

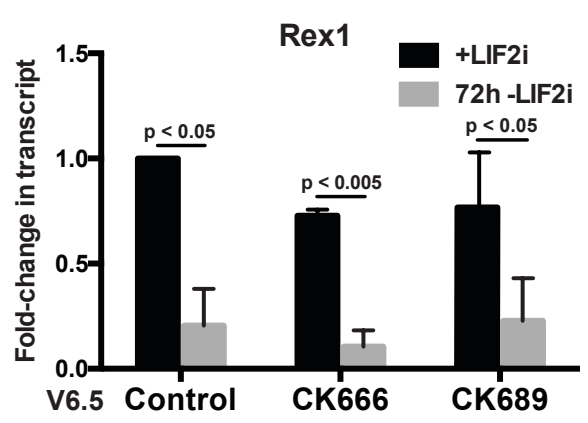
available under aCC-BY-NC-ND 4.0 International license.

$\mathbf{D}$

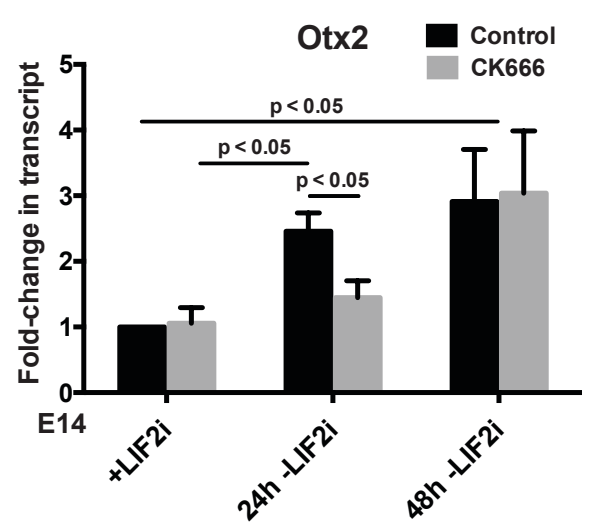

G

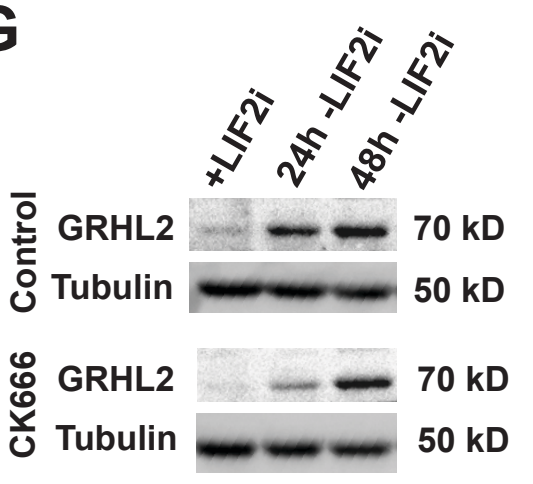

Rex1

+LIF2i

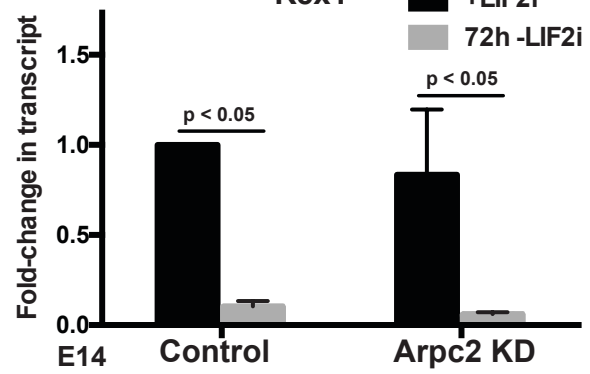

E

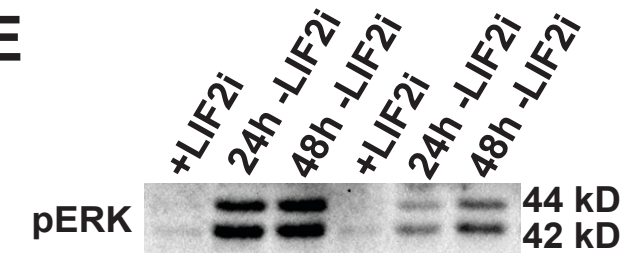

Tubulin $42 \mathrm{kD}$
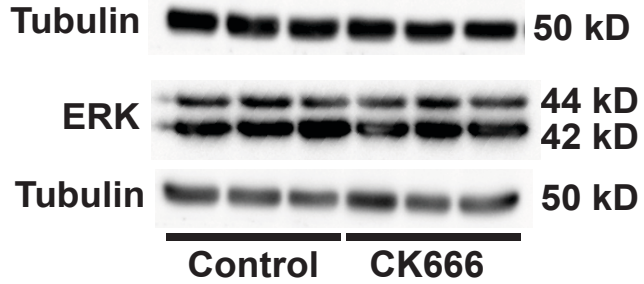

H

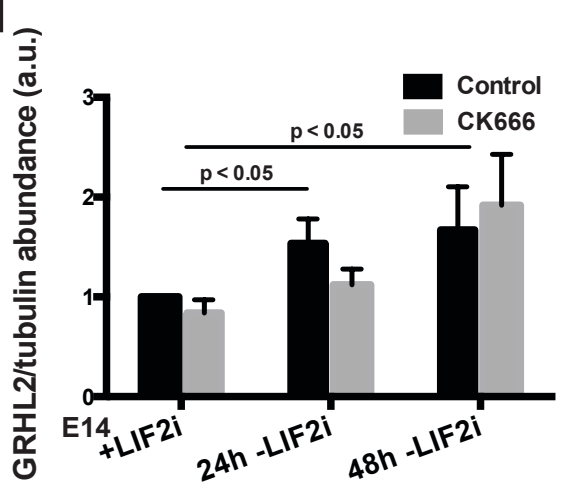

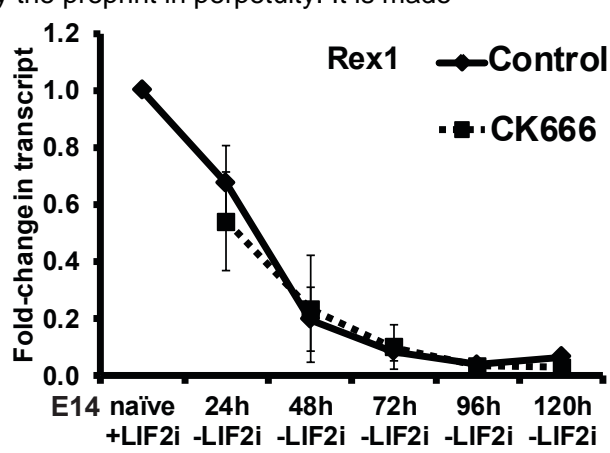
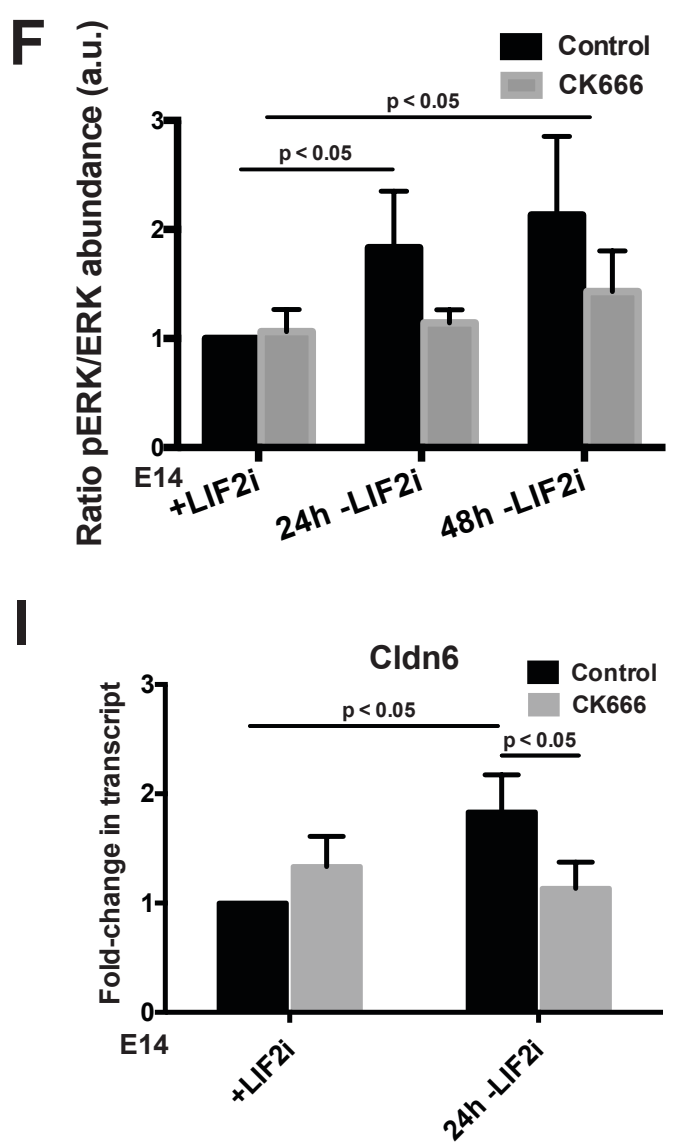

(which was not certified By peer review) is the gauthor/funder. Who has granted bioRxiv a license to display the preprint in perpetuity. It is made
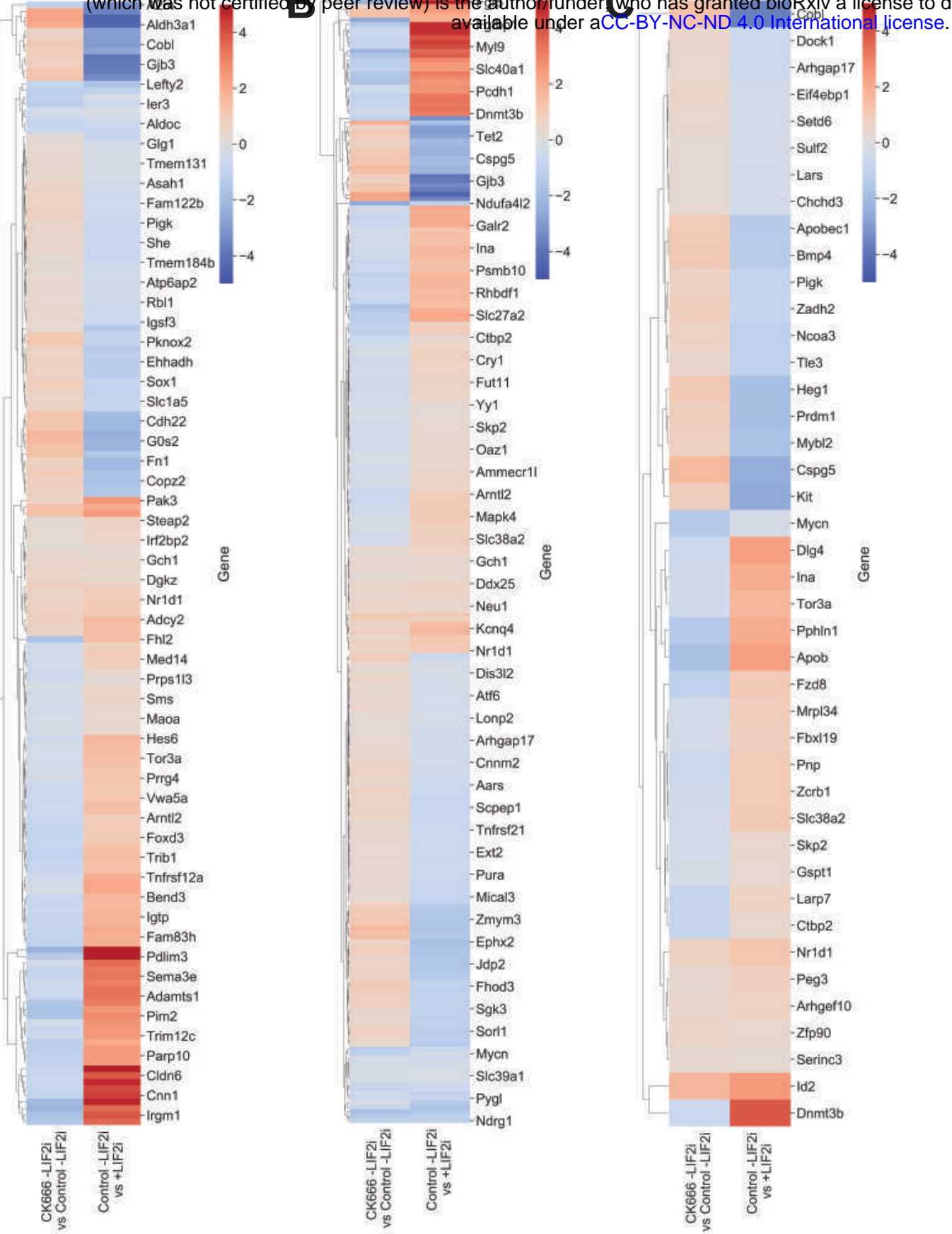

D

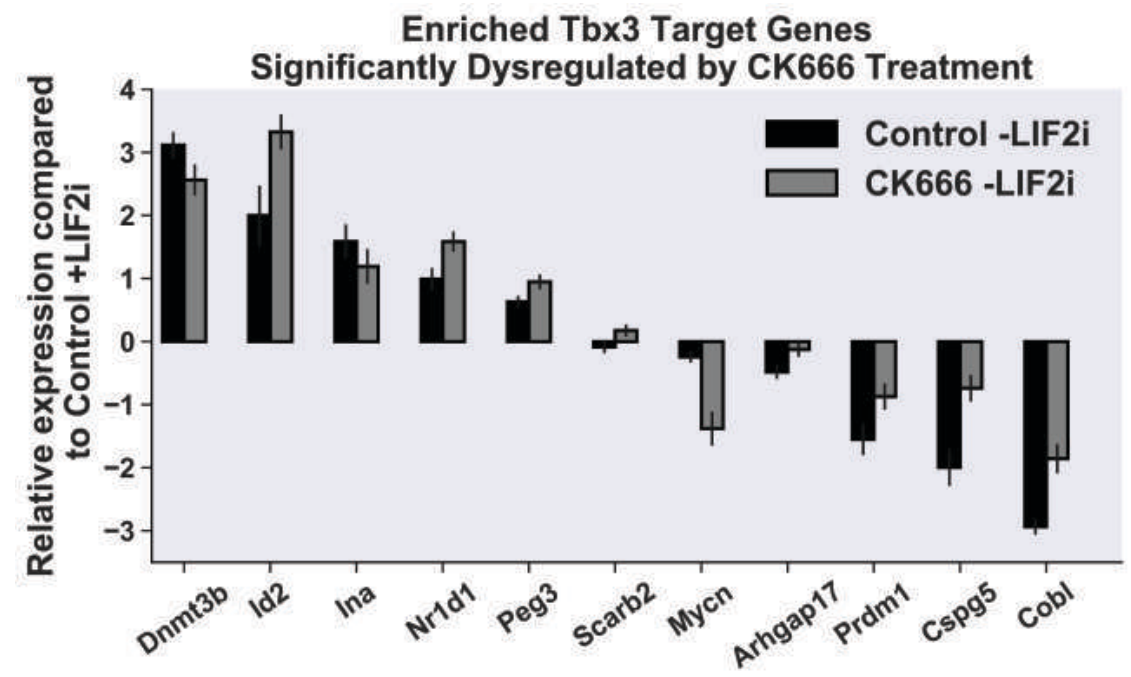

Aloisio and Barber (2021), Fig 5 
bioRxiv preprint doi: https://doi.org/10.1101/2021.11.02.465785; this version posted November 4,2021 . The copyright holder or this preprint
(which was not certified by peer review) is the author/funder, who has granted
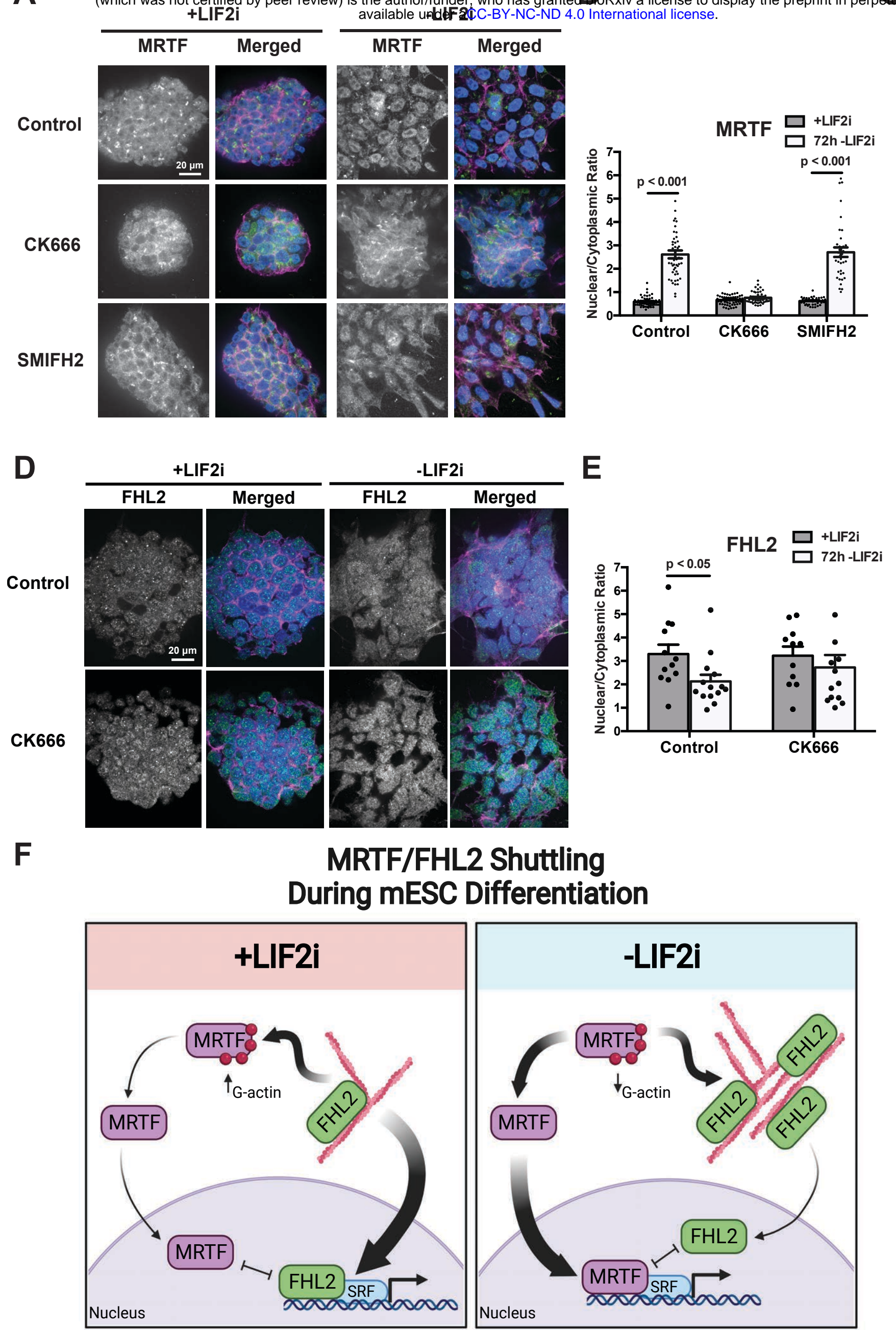

Aloisio and Barber (2021), Fig 6 
bioRxiv preprint doi: https://doisrg/10.1101/2021.11.02.465785; this versjon posted November 4, 2021. The copyright holder for this preprint
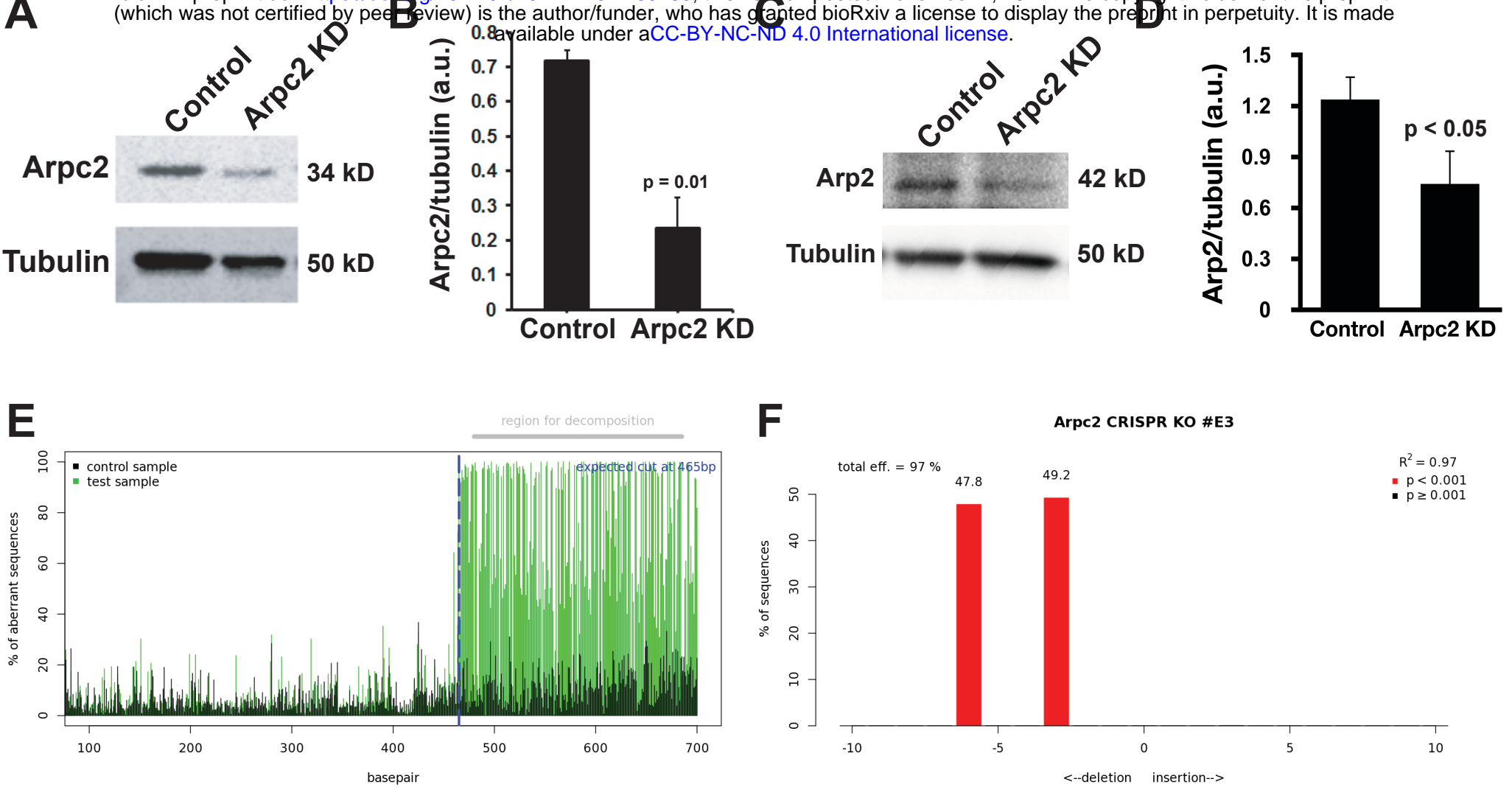

G

H
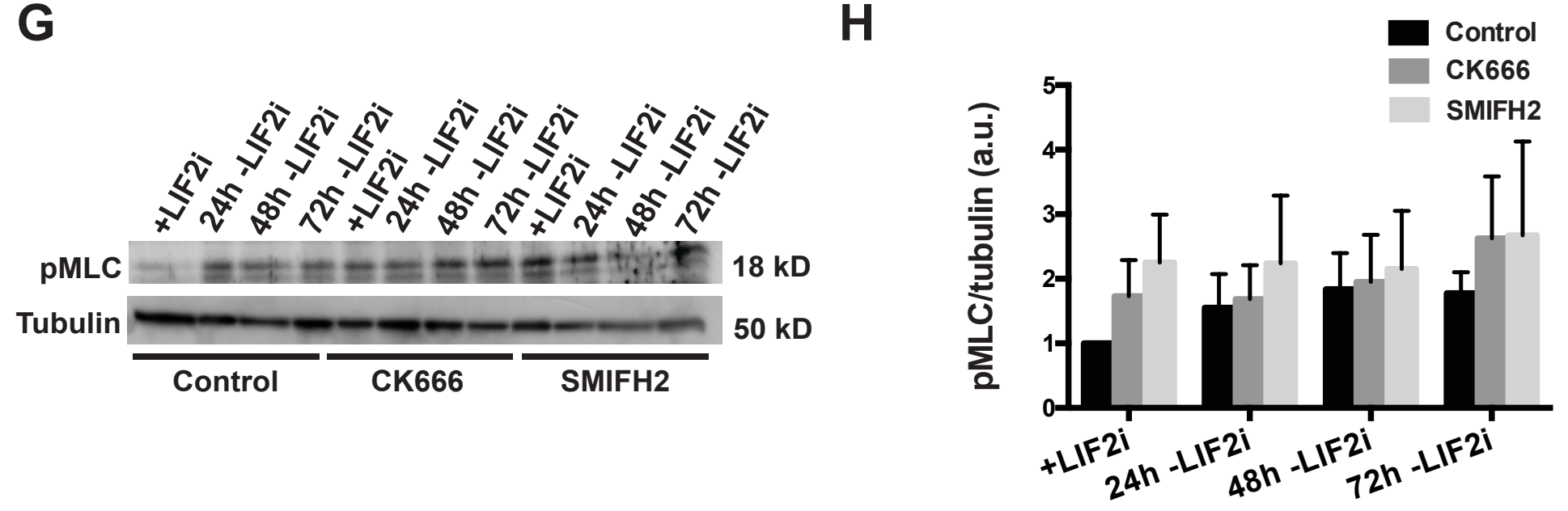

Video S1. E14 cell colony morphology in the presence of LIF2i. DIC time-lapse recording of E14 cells with images taken every $10 \mathrm{~min}$ for $18 \mathrm{~h}$ on gelatin-coated glass, $40 \mathrm{x}$.

Video S2. E14 cell colony morphology at 24-42 h -LIF2i. DIC time-lapse recording of E14 cells with images taken every $10 \mathrm{~min}$ for $18 \mathrm{~h}$ on gelatin-coated glass, $40 \mathrm{x}$. 
bioRxiv preprint doi: https://doi.org/10.1101/2021.11.02.465785; this version posted November 4, 2021. The copyright holder for this preprint A (which was not certified by peer review) is the author/funder, who has grantedioRxiv a license to display the preprintin perpetuity. It is made
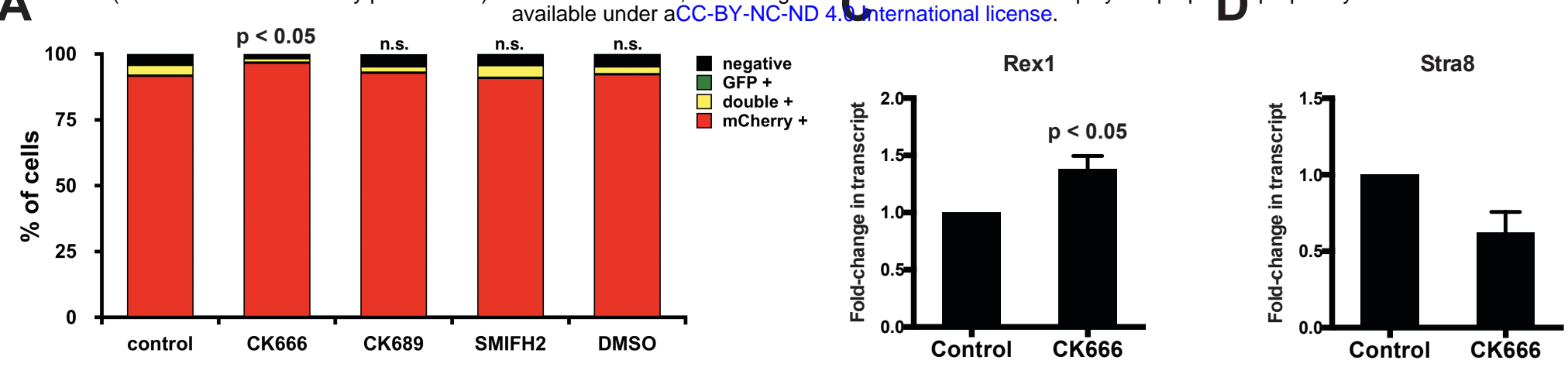

B

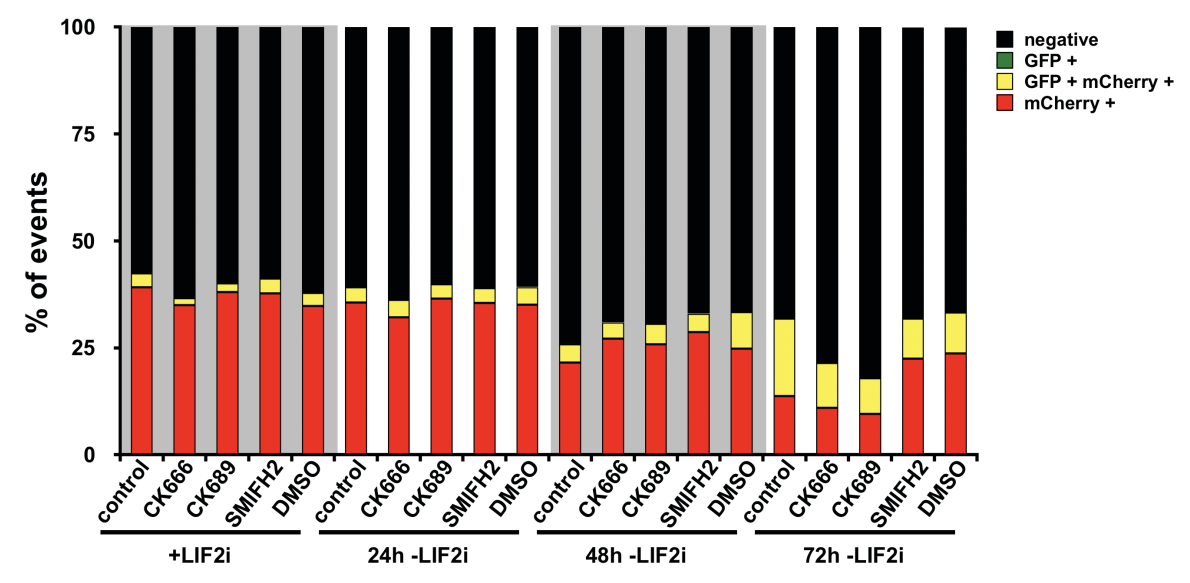

F

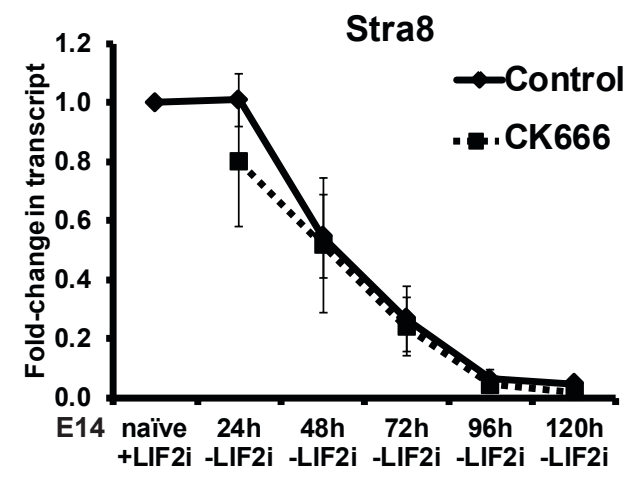

E

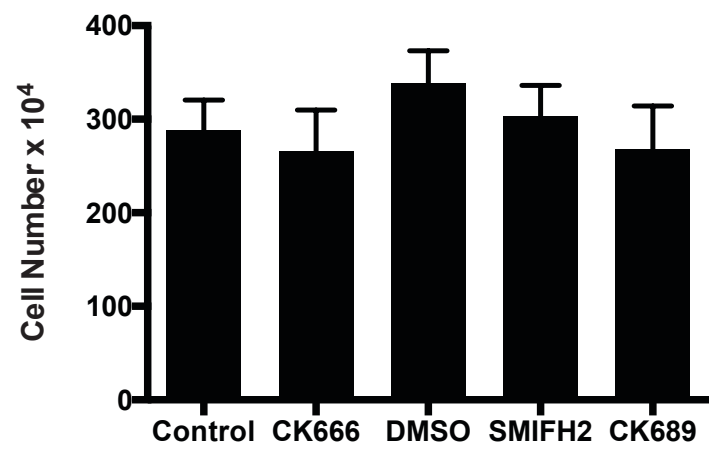


bioRxiv preprint doi: https://doi.org/10.1101/2021.11.02.465785; this version pasted November 4, 2021. The copyright holder for this preprint

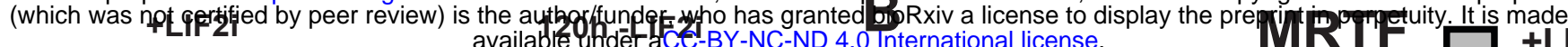
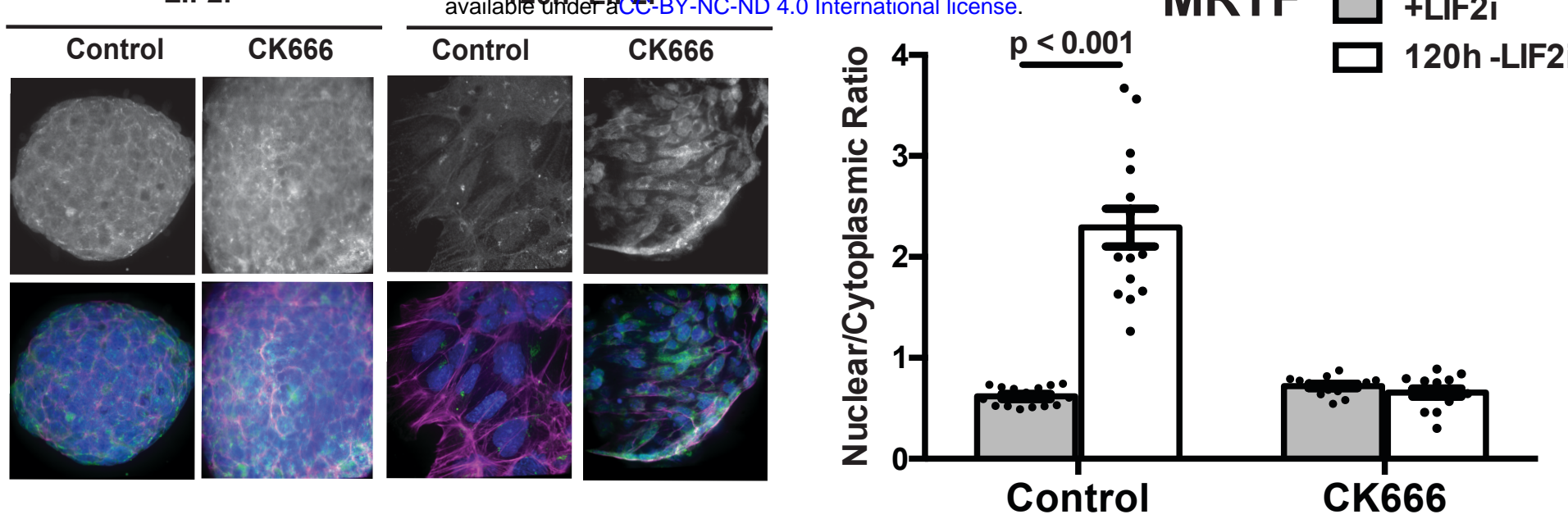